\title{
A Test Protocol for Room-to-Room Distribution of Outside Air by Residential Ventilation Systems
}
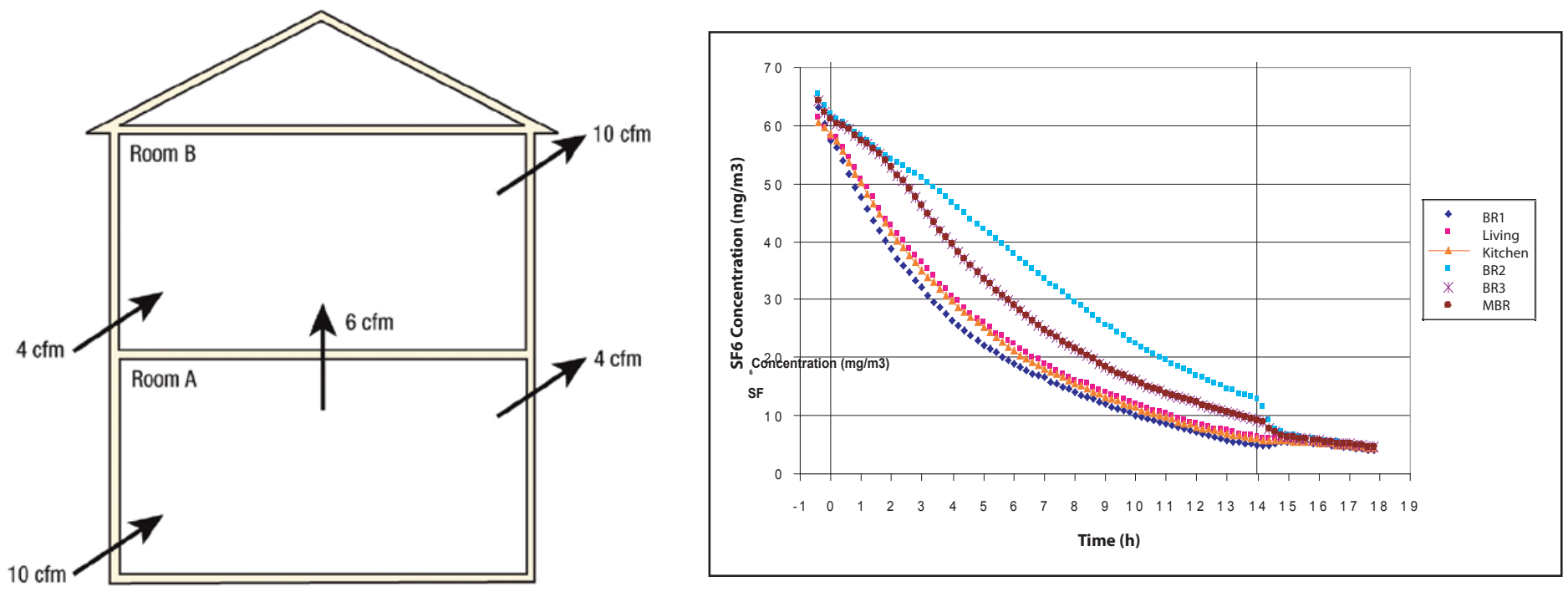

C. Dennis Barley, Ren Anderson, and Bob Hendron

National Renewable Energy Laboratory

Ed Hancock

Mountain Energy Partnership 


\section{A Test Protocol for Room-to-Room Distribution of Outside Air by Residential Ventilation Systems}

Technical Report NREL/TP-550-31548

December 2007

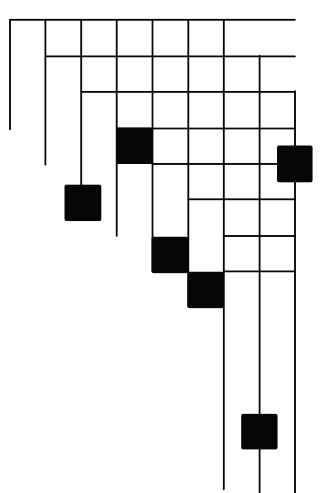

C.D. Barley, R. Anderson, and R. Hendron National Renewable Energy Laboratory

E. Hancock

Mountain Energy Partnership

Prepared under Task No. BET7.8004

National Renewable Energy Laboratory

1617 Cole Boulevard, Golden, Colorado 80401-3393

303-275-3000 • www.nrel.gov

Operated for the U.S. Department of Energy

Office of Energy Efficiency and Renewable Energy

by Midwest Research Institute • Battelle

Contract No. DE-AC36-99-G010337 


\section{NOTICE}

This report was prepared as an account of work sponsored by an agency of the United States government. Neither the United States government nor any agency thereof, nor any of their employees, makes any warranty, express or implied, or assumes any legal liability or responsibility for the accuracy, completeness, or usefulness of any information, apparatus, product, or process disclosed, or represents that its use would not infringe privately owned rights. Reference herein to any specific commercial product, process, or service by trade name, trademark, manufacturer, or otherwise does not necessarily constitute or imply its endorsement, recommendation, or favoring by the United States government or any agency thereof. The views and opinions of authors expressed herein do not necessarily state or reflect those of the United States government or any agency thereof.

Available electronically at http://www.osti.gov/bridge

Available for a processing fee to U.S. Department of Energy and its contractors, in paper, from:

U.S. Department of Energy

Office of Scientific and Technical Information

P.O. Box 62

Oak Ridge, TN 37831-0062

phone: 865.576 .8401

fax: 865.576 .5728

email: mailto:reports@adonis.osti.gov

Available for sale to the public, in paper, from:

U.S. Department of Commerce

National Technical Information Service

5285 Port Royal Road

Springfield, VA 22161

phone: 800.553.6847

fax: 703.605.6900

email: orders@ntis.fedworld.gov

online ordering: http://www.ntis.gov/ordering.htm 


\section{Table of Contents}

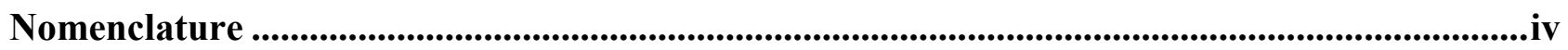

Executive Summary ….......................................................................................................................................

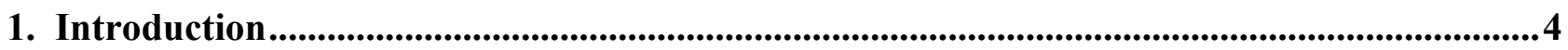

2. Codes and Standards .................................................................................................................................

3. The Need for an Appropriate Metric ......................................................................................................8

4. Comparison of Testing Methods...........................................................................................................12

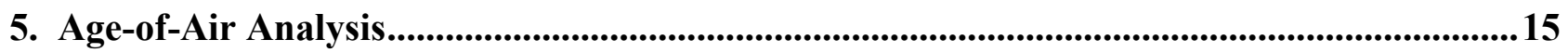

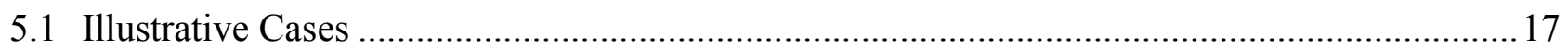

5.1.1 Analytical Example 1: Single Room....................................................................... 19

5.1.2 Analytical Example 2: Two Independent Rooms ........................................................2

5.1.3 Analytical Example 3: Airflow in Series, Room A Smaller..........................................2

5.1.4 Analytical Example 4: Airflow in Series, Room A Larger............................................2

5.1.5 Analytical Example 5: Partial Series Airflow.................................................................2 28

6. The Graph Overlay Method ................................................................................................................31

7. Test and Analysis Protocol ..................................................................................................................33

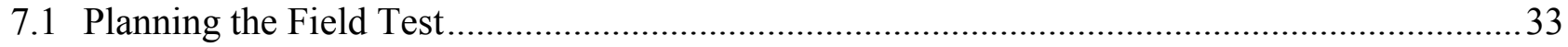

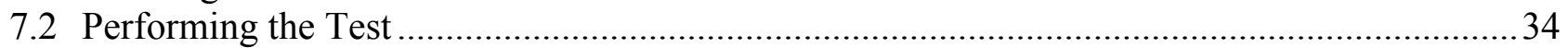

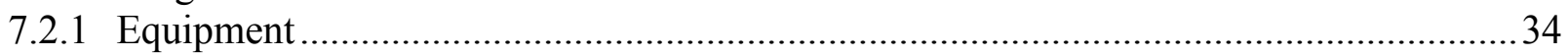

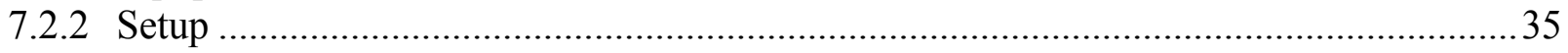

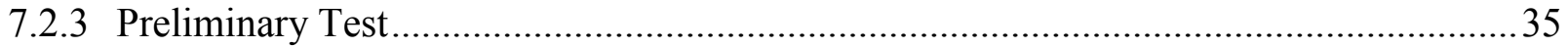

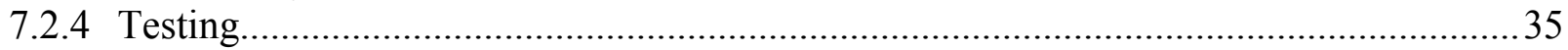

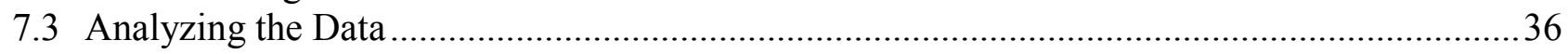

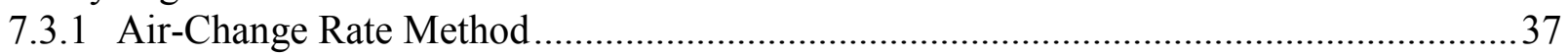

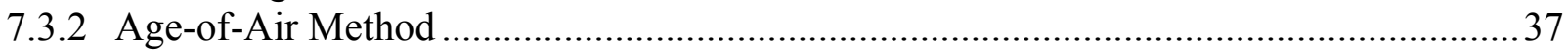

7.4 Special Consideration for Unconditioned Basements ..............................................................

8. Field Test Examples......................................................................................................................39

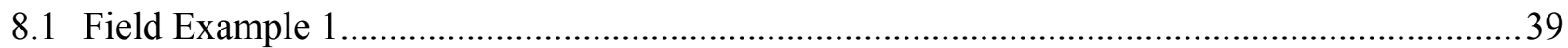

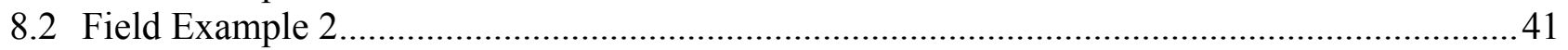

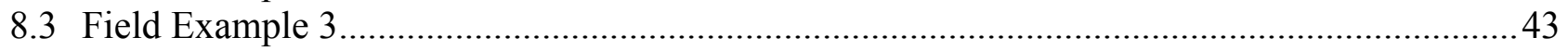

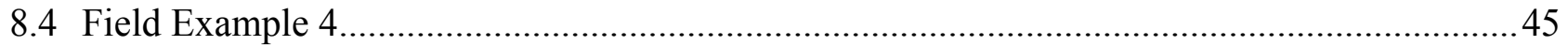

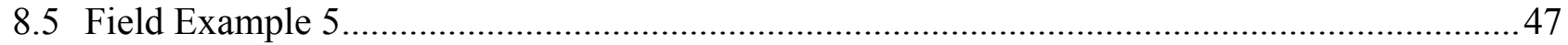

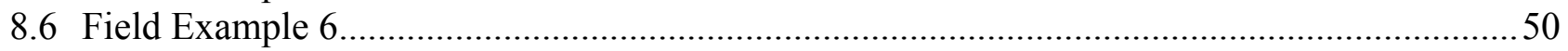

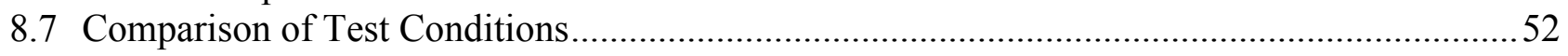

9. Summary and Conclusions ..............................................................................................................5

10. Acknowledgments ...............................................................................................................................54

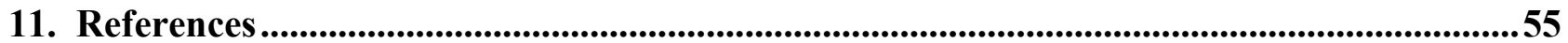




\section{$\underline{\text { Nomenclature }}$}

\begin{tabular}{|c|c|c|}
\hline Symbol & Explanation & Units \\
\hline $\mathrm{A}, \mathrm{B}$ & Room designations in Figs. 1-3 and Eqns. 3-8 (subscripts) & (none) \\
\hline $\mathrm{A}, \mathrm{B}, \mathrm{C}$ & Stages in tracer decay test in Eqns. 28, 30, 31, 32, 35 (subscripts) & (none) \\
\hline $\mathrm{ACR}$ & Air-change rate in a well-mixed building & $\mathrm{hr}^{-1}$ \\
\hline$a, b, d$ & Airflow rates in hypothetical examples & $\mathrm{cfm}$ \\
\hline $\mathrm{ACH}$ & Air changes per hour (unit) & $\mathrm{hr}^{-1}$ \\
\hline $\mathrm{c}$ & Concentration of tracer gas & $\mathrm{lb}_{\mathrm{m}} / \mathrm{ft}^{3}$ \\
\hline $\mathrm{cfm}$ & Cubic feet per min (unit) & $\mathrm{ft}^{3} / \min$ \\
\hline $\mathrm{i}$ & Summation index & (none) \\
\hline$\kappa$ & Decay constant for a well-mixed building & $\mathrm{hr}^{-1}$ \\
\hline$\lambda$ & Decay constant for extrapolated decay curve & $\mathrm{hr}^{-1}$ \\
\hline $\mathrm{m}$ & Mass of tracer gas within a room & $1 b_{m}$ \\
\hline o & Initial condition at start of test (subscript) & $\mathrm{n} / \mathrm{a}$ \\
\hline $\mathrm{p}, \mathrm{q}$ & Constants in Eqn. 8 & (none) \\
\hline RAoA & Reciprocal age-of-air $=1 / \tau$ & $\mathrm{hr}^{-1}$ \\
\hline $\mathrm{T}$ & Elapsed time & $\mathrm{hr}$ \\
\hline $\mathrm{t}$ & Elapsed time & $\min$ \\
\hline$\Delta \mathrm{T}$ & Sampling interval & $\mathrm{hr}$ \\
\hline$\tau$ & Local mean age-of-air & $\mathrm{hr}$ \\
\hline$\tau_{\mathrm{I}}$ & Portion of age-of-air calculated from test data & $\mathrm{hr}$ \\
\hline$\tau_{\mathrm{II}}$ & Portion of age-of-air calculated from extrapolated curve & $\mathrm{hr}$ \\
\hline $\mathrm{V}$ & Room volume & $\mathrm{ft}^{3}$ \\
\hline
\end{tabular}




\section{Executive Summary}

The Building America program, sponsored by the U.S. Department of Energy (DOE), advocates a systems-engineering approach to accelerate the development and adoption of advanced building energy technologies in production housing. Building America partners with crosscutting residential building industry teams to produce advanced homes on a community scale. A schedule of progressive goals over time is defined in terms of percent whole-house source energy savings, ranging from $30 \%$ savings attained in 2007 through the long-term goal of net zero-energy homes by 2020. The program also addresses the comfort and efficiency of various facets of the building design, toward improvements in future projects. Residential building systems are evaluated by conducting successive design, test, redesign, and retest iterations until cost and performance tradeoffs yield innovations that can be implemented in production-scale housing. The National Renewable Energy Laboratory (NREL) provides technical support to the various industry teams, which includes field testing of prototype homes to verify the attainment of energy savings and other program goals.

In striving to increase overall energy efficiency in homes, it is important to meet occupants' indoor air quality (IAQ) needs while minimizing energy usage caused by outside air exchange. Source control is used to eliminate contaminants to the extent possible. Then, well-controlled ventilation is needed to dilute the contaminants that remain. In addition to the amount of outside air provided, the distribution of that air within the home is also important. Nonuniform ${ }^{1}$ distribution of outside air can lead to overventilation in one part of a home and underventilation in another part at the same time. Common examples of nonuniform outside air distribution include natural infiltration in multistory homes and single-point supply or exhaust mechanical ventilation systems. Bedrooms, where occupants typically spend more time than in any other room, are often underventilated because of (1) their location on an upper story of the home, (2) closed doors for privacy or noise buffering, and (3) the lack of a ventilation port such as an exhaust fan. More sophisticated and costly mechanical ventilation systems, such as ducted ventilation or forced-air-integrated ventilation, may significantly improve the distribution of outside air throughout the home. Field testing is needed to verify the performance advantages of such systems in terms of outside air distribution. However, there is no generally accepted or widely used test and analysis protocol for this purpose. Such a protocol is developed in this report.

A significant issue with developing such a test protocol is the need to resolve two conflicting facts regarding occupant behavior:

- IAQ depends on occupant behavior, as it affects the magnitudes and locations of the various pollutant sources within the home, in addition to the airflows generated by a ventilation system.

- At the time of testing, occupant behavior is unknown, because (1) Building America field testing usually occurs in new, unoccupied homes, and (2) several families may occupy the home over its life, and the ventilation system will not be redesigned for each new occupant.

Our approach to resolving this conflict is based on the concept of local mean age-of-air, which is defined as the average time that has elapsed since the air at a particular location has entered the

\footnotetext{
${ }^{1}$ The rationale for using uniformity as a performance criterion is explained in Section 3.
} 
building. Without knowing the actual pollutant distribution in the home (because of unknown occupant behavior), we rely on the concept that the longer the air has been in the home, the more it is likely to be polluted. Age-of-air measurement and analysis, discussed in Section 5, is a wellknown technical approach, which has been characterized as follows in ASHRAE Standard 129 (ASHRAE 2002):

"Where the test method will not be usable in all field situations, it is generally applicable in laboratory test rooms. Future versions of the standard will benefit from additional experience with the test method in the field, perhaps making the test method more widely acceptable."

Specific limitations cited in Standard 129 include:

- Mechanically ventilated test space, with the difference between the maximum and minimum air supply flow rates within $10 \%$

- Limited air infiltration, exfiltration, and air exchange with surrounding indoor spaces.

We have adapted the age-of-air method for use in homes, where infiltration typically contributes significantly to the total outside air exchange rate. Because of fluctuations in the weather, the resulting airflow rates ${ }^{2}$ may not be sufficiently steady to enable age-of-air analysis. We address this issue in our test and analysis protocol, which is described in Section 7, through a three-phased approach:

1. We plan field tests for mild weather, to the extent possible.

2. During the field test, we conduct single-tracer (sulfur hexafluoride) decay testing with multiple sampling points.

3. After the test, we examine the tracer decay data and use engineering judgment to select the appropriate analysis method.

In this third phase, the choices of analysis method are as follows:

A. If the data reveal that the airflows were sufficiently steady and the tracer decay was uniform among the rooms sampled, we calculate the whole-house air-change rate (ACR), in terms of air changes per hour.

B. If the data reveal that the airflows were sufficiently steady and the tracer decay was nonuniform among the rooms sampled, we perform a local mean age-of-air analysis for each room. Because ventilation performance is customarily described in terms of airflow rates and ACRs, in which higher numbers correspond to more air exchange, we use reciprocal age-of-air (RAoA) as our primary metric and a surrogate for IAQ.

C. If the data reveal that the airflows were not sufficiently steady, we use the semiquantitative Graph Overlay Method, described in Section 6, to bracket the RAoA numbers, describe the amount of nonuniformity among the rooms, and in some cases rank the rooms in order of RAoA.

\footnotetext{
${ }^{2}$ The airflows that affect the steady-state criterion include outside air entering the building as well as interzonal airflows, caused by both infiltration and mechanical ventilation.
} 
To illustrate how our adapted age-of-air analysis applies in several airflow scenarios, we present in Section 5 a series of five hypothetical case studies. These examples foster understanding and serve as reference cases for interpreting field test results. In Section 8, we present results from six actual field tests. Each test result demonstrates a particular set of conditions that is typical in this type of testing and provides an example of the engineering judgment needed to choose the appropriate analysis method.

This test and analysis protocol has been developed as a practical approach for measuring outside air distribution in homes. We recommend the use of this protocol within the context of the Building America field testing program. This approach has been used successfully in a number of field tests and has led to significant insights on ventilation design issues. Performance advantages of more sophisticated ventilation systems over simpler, less-costly designs have been verified, and specific problems, such as airflow short-circuiting, have been identified. The lessons learned from this test approach can make a significant contribution to the attainment of the energy conservation and occupant health and comfort goals of the Building America program. 


\section{Introduction}

The Building America program, sponsored by the U.S. Department of Energy (DOE), advocates a systems-engineering approach to accelerate the development and adoption of advanced building energy technologies in production housing. Building America partners with crosscutting residential building industry teams to produce advanced homes on a community scale. A schedule of progressive goals over time is defined in terms of percent whole-house source energy savings, ranging from $30 \%$ savings $^{3}$ attained in 2007 through the long-term goal of net zero energy homes by 2020. The program also addresses the comfort and efficiency of various facets of the building design, toward improvements in future projects. Residential building systems are evaluated by conducting successive design, test, redesign, and retest iterations until cost and performance tradeoffs yield innovations that can be implemented in production-scale housing. The National Renewable Energy Laboratory (NREL) provides technical support to the various industry teams, which includes field testing of prototype homes to verify the attainment of energy savings and other program goals.

In striving to increase overall energy efficiency in homes, it is important to meet occupants' indoor air quality (IAQ) needs while minimizing energy usage caused by outside air exchange. Source control is used to eliminate contaminants to the extent possible. Then, well controlled ventilation is needed to dilute the contaminants that remain. In addition to the amount of outside air provided, the distribution of that air within the home is also important. Nonuniform ${ }^{4}$ distribution of outside air can lead to overventilation in one part of a home and underventilation in another part at the same time, causing an unfortunate combination of poor IAQ and wasted heating or cooling energy. Common examples of nonuniform outside air distribution include natural infiltration in multistory homes, and single-point supply or exhaust mechanical ventilation systems. Bedrooms, where occupants typically spend more time than in any other room, are often underventilated because of (1) their location on an upper story of the home, ${ }^{5}$ (2) closed doors for privacy or noise buffering, and (3) the lack of a ventilation port such as an exhaust fan. More sophisticated and costly mechanical ventilation systems, such as ducted ventilation or forced-air-integrated ventilation, may significantly improve the distribution of outside air throughout the home. Field testing is needed to verify the performance advantages of such systems in terms of outside air distribution. However, there is no generally accepted or widely used test and analysis protocol for this purpose. Such a protocol is developed in this report.

Residences differ from commercial buildings in several ways that affect ventilation design and testing:

- Ventilation requirements in commercial buildings may vary from one area to another within a building, based on space types and occupancy categories such as those defined in ASHRAE Standard 62.1 (ASHRAE 2004). Homes are generally regarded as single-

\footnotetext{
${ }^{3}$ Energy savings are measured relative to a fixed benchmark that is based on standard construction practices in the mid to late 1990s, around the time the program began (Hendron 2006).

${ }^{4}$ The rationale for using uniformity as a performance criterion is explained in Section 3.

${ }^{5}$ In cold weather, thermocirculation, or the "stack effect," draws outside air in near the bottom of a building and exhausts it near the top. Thus, the air reaching the upper level is not as fresh as the air in the lower level.
} 
function areas, with the exception of special spot ventilation requirements for bathrooms, cook stoves, clothes dryers, etc.

- In homes, infiltration typically constitutes a significant portion of the outside air exchange. This rules out certain measurement techniques that apply specifically to mechanically ventilated spaces, which involve tracer gas injection or measurement in air ducts.

- Tracer gas techniques that require initial uniform mixing of the gas throughout the building are practical in homes, although they are generally not practical in commercial buildings. Either the air handler in a forced-air heating system or portable ducted fans may be used for this purpose.

For these reasons, along with the context of the Building America residential field testing program, the test and analysis protocol presented in this report is specifically designed for use in testing homes.

For consistency, we adopt the following terminology from the ASHRAE Handbook-Fundamentals (ASHRAE 2005):

- "Mechanical ventilation" is the intentional movement of air into and out of a building using fans and intake and exhaust vents.

- "Infiltration" is the flow of outdoor into a building through cracks and other unintentional openings and through the normal use of exterior doors for entrance and egress. (In our test protocol, outside doors remain closed for the duration of the test.)

- "Air exchange" and "outdoor air" (or outside air) include both mechanical ventilation and infiltration.

The ASHRAE definition of ventilation includes mechanical ventilation and intentional natural ventilation, as through windows, but not infiltration. Here, we also use the term ventilation in reference to the overall design for air exchange, which may include allowances for infiltration. We also use the term overventilation in reference to excessive air exchange, and the term underventilation for inadequate air exchange, regardless of the delivery mechanism. This test and analysis protocol measures total air exchange, including mechanical ventilation and infiltration. Throughout this report, all discussion refers to the dilution ventilation approach, as distinct from displacement ventilation.

This report is organized as follows:

- Section 2 discusses domestic and international ventilation standards and codes, some of which address outside air distribution within homes and some of which do not.

- Section 3 discusses our need for a metric to compare outside air distribution in various ventilation systems, the criteria we recognize as important in the selection of a metric, and our choice of reciprocal age-of-air (RAoA) for this purpose.

- Section 4 discusses and compares established multi-zone tracer gas testing methods, including (1) single-tracer, constant injection, (2) multiple-tracer, and (3) single-tracer decay with multiple sampling points (our choice). 
- Section 5 discusses our adaptation of the established local mean age-of-air analysis method, to measure room-to-room differences in outside air distribution, as distinct from air distribution within a room. This section includes a series of five analytical examples that illustrate the relationship between airflow patterns, tracer decay curves, and age-of-air calculations.

- Section 6 presents an alternative, semiquantitative analysis method, which we call the Graph Overlay Method, for use when test conditions are not sufficiently steady to enable age-of-air analysis.

- Section 7 outlines the step-by-step process for field test planning, measurement, and data analysis.

- Section 8 shows and discusses results of six actual field tests that illustrate the test and analysis protocol under a variety of typical test conditions.

- Finally, conclusions, acknowledgments, and references are presented in Sections 9, 10, and 11 , respectively. 


\section{Codes and Standards}

Some ventilation codes and standards address the issue of room-to-room outside-air distribution; others do not. For example, the ASTM Standard Guide for Specifying and Evaluating Performance of Single Family Attached and Detached Dwellings-Indoor Air Quality (ASTM 2004) includes the following recommendations, in Table X1.2:

"The outdoor ventilation air should be distributed throughout the occupied portions of the building to provide adequate ventilation to all occupied spaces."

"There should be an identifiable means of providing outdoor air to each occupiable room."

"Measured or calculated outdoor airflow rates into the room should correspond to a specified air change rate in units of air changes per hour or $\mathrm{L} / \mathrm{s}(\mathrm{cfm})$ per occupant."

Residential ventilation standards in numerous European countries, including Belgium, Denmark, Finland, Germany, Italy, Netherlands, Norway, Sweden, and the United Kingdom, specify minimum ventilation requirements in bedrooms, living rooms, or both (McWilliams and Sherman 2005). Canadian standard CAN/CSA-F326-M91 (CSA 2003) requires: "Ventilation systems shall be designed so that ventilation air is circulated throughout the occupied zone." In the United States, ASHRAE Standard 62.2-Ventilation and Acceptable Indoor Air Quality in Low-Rise Residential Buildings (ASHRAE 2007) - specifies a minimum whole-house mechanical ventilation rate that depends on the timing of ventilation air delivery; however, it is silent about the distribution of outside air. The Washington State Ventilation and Indoor Air Quality code (WAC 2004) is also silent about outside air distribution. The Minnesota building code (MN Chapter 7672) requires: "Outdoor air must be delivered to each habitable room by individual inlets, separate duct systems, or a forced air system." The codes and standards that require outside air distribution in homes emphasize the importance of having a test protocol to verify this aspect of ventilation system performance. 


\section{The Need for an Appropriate Metric}

To consistently measure and compare the performance of various residential ventilation systems, we need a well-defined metric that quantifies room-to-room differences in the supply of outside air. To serve the purposes of the Building America field testing program, the metric and the associated measurement protocol should have the following qualities:

- The metric should indicate differences in outside air distribution from room to room within a home.

- The metric should be measurable in a routine field test of a prototype home.

- The measurement protocol should be practical in terms of the required equipment, technician expertise, equipment calibration, test conditions, setup time, testing time, cost, etc.

- The metric should be independent of occupant behavior.

We stipulate that the metric should be independent of occupant behavior for two reasons:

- Building America field testing usually occurs in new, unoccupied homes. At that time, there is no occupant behavior, and future occupant behavior is unknown.

- Various families may occupy the home over its life, and the ventilation system will not be redesigned for each new occupant.

However, IAQ does depend on occupant behavior, as well as on airflows. To illustrate, Figure 1 shows a hypothetical example involving two rooms, A and B.

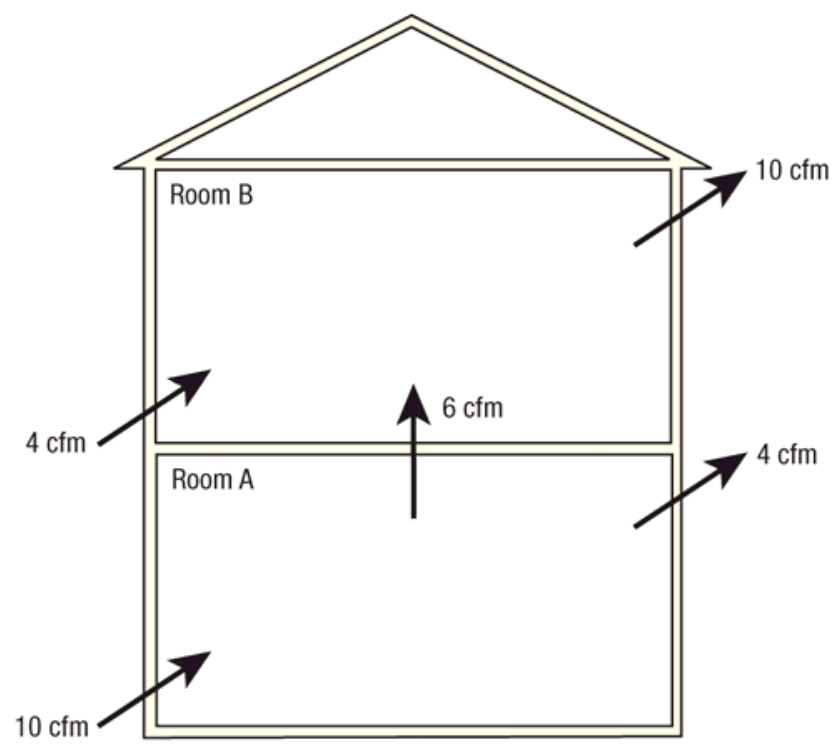

Figure 1. Hypothetical airflow pattern in two rooms 
Each room receives some outside air directly and exhausts some air to the outside. In addition, there is interzonal airflow from Room A to Room B. Each room receives a total airflow of $10 \mathrm{cfm}$. However, Room A receives $10 \mathrm{cfm}$ of outside air directly, while Room B receives $4 \mathrm{cfm}$ of direct outside air and $6 \mathrm{cfm}$ of interzonal air from Room A. How can we compare the air exchange in these two rooms? Figure 2a shows one possible occupant behavior scenario, with a cigar smoker in Room B. Both the $4 \mathrm{cfm}$ of direct outside air and the $6 \mathrm{cfm}$ of interzonal air help to dilute the smoke. Thus, it might seem that the effective ventilation rate (in terms of pollutant dilution) is 10 $\mathrm{cfm}$, as it is in Room A. However, Figure $2 \mathrm{~b}$ shows a different occupant behavior scenario, with the cigar smoker moved to Room A. In this case, the interzonal airflow has a negative impact on Room B. The effect of this airflow is clearly not the same as would be provided by $10 \mathrm{cfm}$ of direct outside air entering Room B. This example illustrates how the value, or detriment, of interzonal airflow depends on occupant behavior.

In our testing situation, with occupant behavior unknown, we can consider the typical locations of pollutants, in addition to the cigar-smoker example, in a home:

- Bathrooms and kitchen ranges are often strong pollutant sources. However, these locations are generally treated with spot exhaust ventilation. The ventilation system should be designed so these pollutants do not flow to other portions of the home.

- A new carpet or new paint in a new or remodeled home may outgas for a period of time. These sources are distributed throughout most of the home.

- Household cleaning products that emanate vapors are used on hard floors, carpets, furniture, windows, etc., throughout the home.

- A cat litter box, often a strong odor source, may be located at any of numerous locations in the home, unknown at the time of testing.

- Hobby activities involving the use of glue, paint, varnish, etc., may be used at any of numerous locations in the home, unknown at the time of testing.

- Human and pet body odors emanate from sources that move about the home.

- Perfume, cologne, hairspray, and other personal grooming products that emit vapors may be used in bathrooms, bedrooms, or elsewhere in the home.

Thus, every part of the home is a potential pollution source location. One generalization we can make is that the longer air has been in the home, the more it is likely to be polluted. This leads us to the concept of age-of-air, which is defined as "the average time elapsed since molecules of air in a given volume of air entered the building from outside" in ASHRAE Standard 129 (ASHRAE 2002). For a given rate of pollution in a well-mixed home, newer air is fresher (higher quality) and older air is staler (lower quality). When the rate of pollution and the locations of pollutants are unknown, as they are in our testing situation, we can generalize that older air is likely to be staler, and newer air is likely to be fresher. This is the basis of our metric. In Section 5, we develop the concept of age-of-air $(\tau)$ for use in our test protocol. Because ventilation performance is customarily described in terms of airflow rates (in $\mathrm{cfm}$ ) and air-change rates (ACRs, in air changes per hour $[\mathrm{ACH}])$, in which higher numbers correspond to more air exchange, we use RAoA, $1 / \tau$, as our primary metric. RAoA is a measure of air freshness, qualified by the uncertainties about pollutant strength and location inherent in our testing situation. In the special case of a single wellmixed enclosure, RAoA is equal to ACR. 


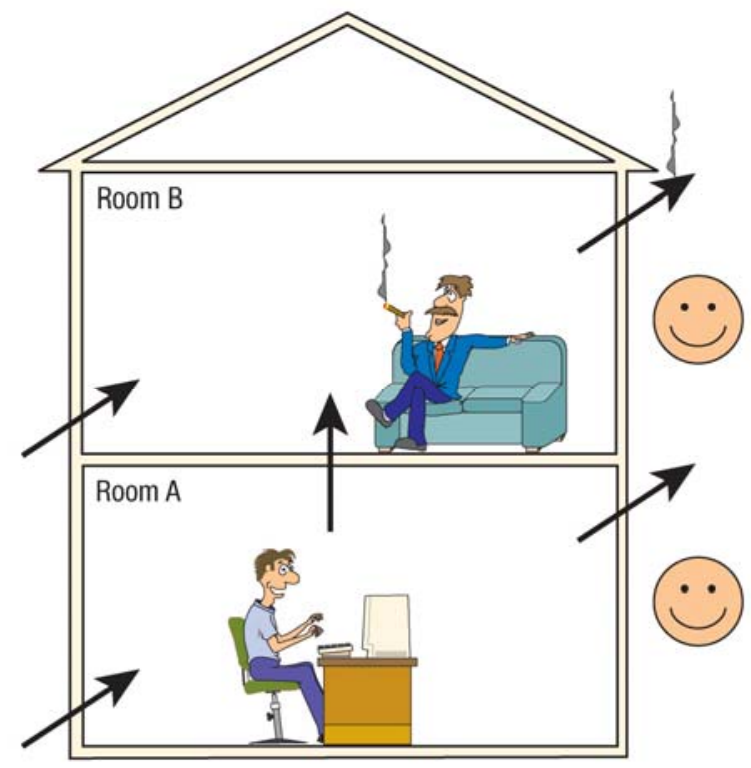

(a) Pollutant source in Room B

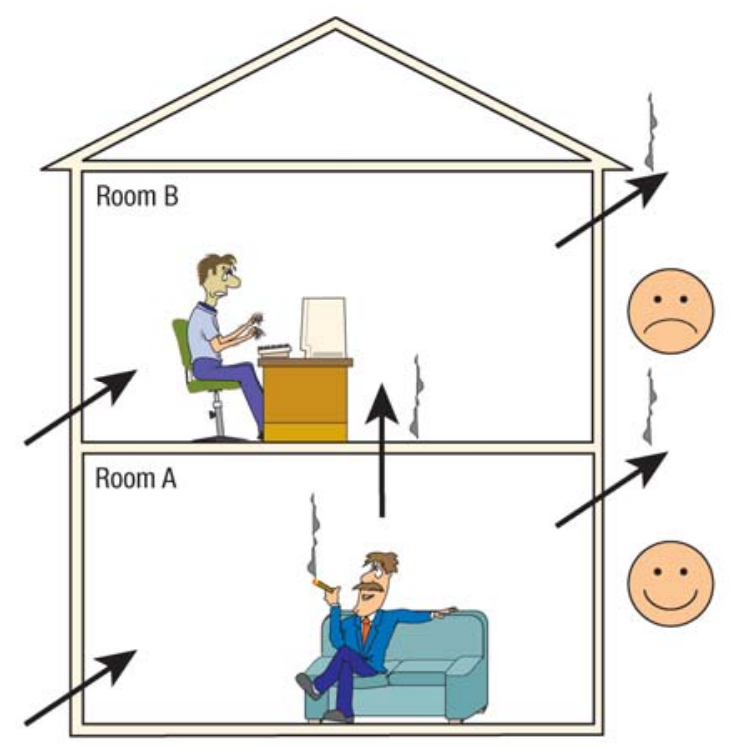

(b) Pollutant source in Room A

Figure 2. Comparison of occupant behavior in two cases 
Returning to Figure 1, where the airflows are indicated but occupant behavior is unknown, we can now evaluate the difference in air exchange between the two rooms based on the airflow pattern. Room A receives $10 \mathrm{cfm}$ of direct outside air, which is presumably unpolluted. Room $\mathrm{B}$, in addition to $4 \mathrm{cfm}$ of direct outside air, receives $6 \mathrm{cfm}$ of interzonal air that we deem to be less fresh. This air has been in the building for some time, so it may convey some pollution when it arrives. Using the age-of-air concept and assuming each room is well mixed, the age-of-air is $1.67 \mathrm{hr}$ in Room A and $3.00 \mathrm{hr}$ in Room B. Thus, the RAoA is 0.60/hr in Room A and 0.33/hr in Room B, reflecting the dissimilarity in expected air freshness. (The calculations of these values are presented in Section 5, Analytical Example 5. The volumes of Rooms A and B are assumed to be 1,000 $\mathrm{ft}^{3}$ and 1,200 $\mathrm{ft}^{3}$, respectively.) We are, in a sense, hedging our bets about the location of the cigar and the distribution of other pollution sources in the home. If we had cigars in both rooms, RAoA would indicate the dilution of the smoke and thus the air quality in each room. The air in room A would be fresher, as indicated by the higher value of RAoA. Of course, we can consider hypothetical pollution scenarios that produce air quality patterns that are skewed from the RAoA metric (such as Figure 2a). However, an alternative scenario with the opposite effect (such as Figure $2 b$ ) can always be considered. The use of RAoA as a metric for air freshness corresponds to the assumption that pollution sources are equally likely to be located anywhere in the home. Although not proven with actual field data, we use this assumption as our best estimate of the occupant behavior that is unknown at the time of testing.

This study of room-to-room variations in outside air distribution is distinct from the issue of how much of the outside air within a room reaches the location of the occupants, referred to as ventilation effectiveness in ASHRAE Standard 129 (ASHRAE 2002). In the test protocol described in Section 7, we use small electric mixing fans in each room to establish a well-mixed condition to eliminate variations within a room from our measurements. 


\section{Comparison of Testing Methods}

Methods of testing multizone air exchange rates with single and multiple tracer gases are discussed by Dietz et al. (1986), Fortmann et al. (1990), Harrje et al. (1985 and 1990), Sherman and Dickerhoff (1989), Sinden (1978), and Wortman et al. (1982). Techniques include:

- Constant-concentration, single-tracer tests are used to determine rates of outside air entering each zone directly (see Fortmann et al. 1990 and Harrje et al. 1985, 1990).

- Multiple-tracer tests are used to determine the matrix of airflow rates among the various zones and the atmosphere. These methods include the "multi-tracer measurement system" (MTMS) described by Sherman and Dickerhoff (1989) and the passive perfluorocarbon tracer (PFT) method described by Dietz et al. (1986).

- Single-tracer (often sulfur hexafluoride $\left[\mathrm{SF}_{6}\right]$ ) decay tests with multiple sampling points may be used to determine local mean age-of-air (ASHRAE 2002; Grieve 1991; Rudd and Lstiburek 2000).

Under the heading "Examples of Indoor Air Quality Performance Statements," the ASTM Standard Guide for Specifying and Evaluating Performance of Single Family Attached and Detached Dwellings -Indoor Air Quality (ASTM 2004) comments:

"Outdoor airflow rates can be measured for individual rooms using multizone tracer gas techniques." "Similarly, age of air measurements using tracer gas (based on ASHRAE Standard 129) could be used."

"Multizone tracer gas techniques have not been standardized and are generally used only in research, and age of air measurement [is] still in the realm of research."

Reardon and Shaw (1997) used a multizone, single-tracer gas decay method to test and compare a variety of ventilation systems in homes without forced-air heat. They do not define a metric for room-to-room differences in outside air distribution. However, they do conclude "the local exhaustonly strategy... [does] not provide adequate air distribution to the various individual rooms," and "the rooms on the lower stories receive almost all of the outdoor air." In contrast with this conclusion, a modeling study of manufactured (one-story) homes by Persily and Martin (2000) shows reasonably good air distribution among rooms with a single-point exhaust fan. (The ratio of the room-to-room maximum to minimum ACRs ranges from 1.3 to 1.6 in a variety of cases.)

With the constant-concentration, single-tracer test method, a single tracer gas in injected into each zone of a building using feedback controllers that regulate the gas injection rate in each zone to maintain the same target ${ }^{6}$ concentration throughout the building. The resulting rates of gas injection in each zone correspond to the amounts of air entering each zone directly from outside the building. Interzonal airflow has no effect on the measurement, because it has the same concentration of tracer gas as the air already in the zone. If this test were applied to the hypothetical scenario shown in Figure 1 (Section 3), the test would indicate $10 \mathrm{cfm}$ in Room A and $4 \mathrm{cfm}$ in Room B, but it would not detect the $6 \mathrm{cfm}$ of interzonal airflow. Such a result answers the question, "How much outside air enters each room directly?" although it does not answer the question, "What is the net effect of all the airflows, including interzonal airflow, on diluting

\footnotetext{
${ }^{6}$ The actual concentration varies somewhat with time, because of the response time of the control system.
} 
pollutants in each room?" The test results are sensitive to the calibration of the test equipment. For example, if the measurement of an injection rate is high by $10 \%$ or $20 \%$, the indicated airflow rate will be high by the same percentage. Also, differences in calibration from one zone to another would give false indications of the amount of nonuniformity among the zones.

With the multiple-tracer gas method described by Sherman and Dickerhoff (1989) (the MTMS method), a different tracer gas is injected into each zone with a constant target concentration, and all the tracer gases are detected in each zone. Analysis of the measurements, based on a linear set of differential equations and assumed well-mixed conditions in each zone, indicates the matrix of all airflows among the zones and between each zone and the outdoors. Applied to the scenario shown in Figure 1, this test would indicate the $10 \mathrm{cfm}$ of outside air entering Room A, the $4 \mathrm{cfm}$ of outside air entering Room B, and the $6 \mathrm{cfm}$ of interzonal airflow from Room A to Room B. This is a complete set of information about the airflow pattern. Such a result is useful for diagnosing specific airflow problems. Also, if the locations and emission rates of all the pollutants are known, this method can be used to predict air quality in each zone. In the Building America testing scenario where occupant behavior is unknown, an assumption about pollutant distribution would be needed to relate the results to IAQ. If we were to assume that pollutants are uniformly distributed over the volume of the home, the IAQ indicated by this method would correspond to the concept of air freshness based on RAoA. However, the multiple-tracer measurement procedure is more complex and expensive than the age-of-air method described in Sections 5 and 7. As with the constant-concentration, single-tracer test, the multiple-tracer test results are sensitive to the calibration of the test equipment. Also, the mathematical operation of relating tracer gas measurements to airflow rates may significantly magnify measurement errors and errors introduced by weather instability, leading to higher uncertainty in the results.

The multiple-tracer PFT method described by Dietz et al. (1986) enables multiple-tracer testing of up to six zones with little on-site equipment and low expense, over periods ranging from hours to years. A different type of tracer gas is continuously injected into each zone via a small passive source, or emitter capsule. Capillary adsorption tube samplers (CATS), placed in each zone, detect the average concentrations of each tracer in the zone. Analysis of the CATS at the Brookhaven National Laboratory yields the matrix of average ${ }^{7}$ airflow rates among all the zones and the atmosphere. Theoretically, this test would yield the same results as the MTMS method for the same test conditions and test duration. The PFT method has the advantage of being relatively unobtrusive in the home, allowing testing for long periods while the home is occupied. Thus, the effects of occupant behavior, as well as averaging over a variety of weather conditions, can be included in the measurement. It is also an inexpensive way of diagnosing airflow patterns, such as entrainment of pollutants from a garage (auto fumes) or a crawl space (radon gas). However, accuracy is a significant issue with the PFT method. Sources of error include laboratory instrument calibration and contamination of the CATS samplers. Palmiter and Bond (1991) have compared PFT measurements with concurrent measurements using the MTMS method. One issue noted in that study is a bias caused by reciprocal averaging. Because ventilation rates are inversely related to tracer concentrations, the measurement of time-averaged tracer concentrations systematically underestimates the average ventilation rate when the rate varies significantly during the test. When evaluating ventilation systems, the reciprocal average ACR is regarded as more meaningful than the mean ACR, because it more closely corresponds to the exposure of occupants to pollutants over the time period. (For heating and cooling calculations, the actual average ACR is more meaningful.)

\footnotetext{
${ }^{7}$ Tracer gas concentrations are averaged over time, leading to reciprocal averages of the airflow rates.
} 
Palmiter and Bond also report that "Due to unexplained difficulties with the PFT sampler analysis at Brookhaven, all of the determinations for one of the PFT tracers (oc-PDCH) were grossly in error." At the one test site where oc-PDCH was not used, "The PFT test . . gave flows which were in very good agreement with the MTMS measurements." Errors may be larger in short-term PFT tests than in longer-term tests, because of the lower signal-to-noise ratio in the CATS samplers. An additional disadvantage of the PFT method is the delay in obtaining results based on analysis at a remote laboratory.

The single-tracer decay, multiple sampling points test begins with injecting a tracer gas (most commonly $\mathrm{SF}_{6}$ ) and mixing it uniformly throughout the home. Then, injection and mixing cease and a single gas analyzer with tubing to each sampling point is used to monitor the concentration of the tracer gas at several locations as it decays. The age-of-air can be calculated based on the decay curve at each measurement point, provided the test conditions are sufficiently steady. If applied to the scenario shown in Figure 1, this test would indicate the RAoA in each room (assuming a fully mixed condition in each room, which we try to achieve in our test protocol). It would not indicate any of the specific airflows - neither the $10 \mathrm{cfm}$ in Room A, the $4 \mathrm{cfm}$ in Room B, nor the $6 \mathrm{cfm}$ of interzonal airflow. However, the specific airflows are not needed to determine the metric we have chosen for our test protocol. Because tracer decay rates are determined from relative tracer gas concentrations, the results of the decay test are not sensitive to the calibration of the gas analyzer, provided that it is linear and that the gas concentration is in an appropriate range for the test. (A zero-offset calibration error would affect the results, but this is easy to check in the field by observing the measurement before any tracer gas is introduced.) This test and analysis protocol is developed in Sections 5 and 7.

Wortman et al. (1982) explored a different analytical method with the single-tracer decay, multiplesampling-point test. They applied the tracer decay data to the linear system of differential equations describing the matrix of interzonal airflows. They concluded, "Preliminary results indicate that zone-by-zone infiltration rates can be derived using a single tracer gas, but interzonal rates are generally not well determined." This is consistent with the scope of the constant-concentration, single-tracer test method described above. 


\section{Age-of-Air Analysis}

Age-of-air is a well-established technical approach that is described by Sandberg (1981), drawing on chemical engineering practice as well as several foreign language ventilation references. Sandberg's description of the concept includes the following statements:

"The 'freshness' of the air and its dilution capability at a particular point is characterized by its 'age.' The age of the air at the point is defined as the time, $\tau$, that has elapsed since the air entered the room."

"The 'younger' the air the better is its dilution capability."

"A measure of the local ventilation rate...can be defined... as [reciprocal age of air]...The local ventilation rate is a measure of the exchange of input air or 'fresh' air at the actual point. [These] definitions... are those normally found in chemical engineering."

Grieve (1991) describes and illustrates age-of-air measurement using pulsed injection, concentration growth, and concentration decay tracer gas methods. He comments, "The concentration-decay method is...the only usable method where a space is naturally ventilated." Grieve provides the following formula for calculating local mean age-of-air at an arbitrary measurement point within an enclosure (nomenclature adapted for this report; see Nomenclature section):

$$
\tau=\frac{1}{c_{o}} \int_{0}^{\infty} c(T) d T
$$

ASHRAE Standard 129 (ASHRAE 2002) describes an age-of-air test and analysis protocol under test conditions that include the following:

- Mechanically ventilated test space, with the difference between the maximum and minimum air supply flow rates within $10 \%$

- Limited air infiltration, exfiltration, and air exchange with surrounding indoor spaces.

Standard 129 states further: "While the test method will not be usable in all field situations, it is generally applicable in laboratory test rooms. Future versions of the standard will benefit from additional experience with the test method in the field, perhaps making the test method more widely acceptable." A primary concern in homes is weather-driven infiltration that is often a significant portion of the total ventilation rate. If the outdoor temperature or wind speed varies significantly during the test period, the flow rate consistency requirement may not be met.

For use in the Building America field testing program, we have adapted the concentration decay, local mean age-of-air method described by Grieve. The entire volume of the home is our test space. Of course, the concentration decay cannot be measured at every point in the home to describe outside air distribution. Our test equipment (described in Section 7) enables sampling at six locations, which is a manageable number of data sets to analyze for each field test. We locate the sampling points in various rooms, and we mix the air within each room so the measurement point represents the entire room. This eliminates the variation of outside air distribution within each room, which is the subject of a separate type of analysis. Thus, we have created a special case of 
the local mean age-of-air test described by Grieve, in which the test space is partitioned into wellmixed zones.

This test protocol relies on the following assumptions:

1. The concentration of tracer gas in the outside air entering the building is zero. This should not be a problem unless exhaust and inlet openings are close to each other.

2. Air exchanges with enclosures that have not been dosed with tracer gas, such as closets, cabinets, and attics, are negligible. Such air exchanges can be minimized by either opening the enclosures to include them in the dosing, or making sure they are well sealed, as with tape. Special consideration for unconditioned basements is discussed in Section 7.4.

3. The rooms in which sampling occurs are well mixed. We use small portable fans to create the mixing, as described in Section 7. We occasionally check the uniformity of tracer concentration within the room by placing several sampling points in the same room (see Section 8, Field Example 1).

4. The effect of the room mixing fans on the room-to-room air distribution is negligible compared to the differences we are measuring. Further research is needed to determine the validity of this assumption. If it is not true, then this test protocol systematically underestimates the nonuniformity of air distribution among rooms.

5. The boundary conditions are steady state. In homes, weather-driven infiltration is always present. This test protocol measures the combined effect of mechanical ventilation and infiltration. To satisfy the requirement for steady-state conditions, we try to schedule field tests during favorable weather conditions, which consist of the following:

- Low wind speeds, to avoid significant wind-driven infiltration, which tends to fluctuate rapidly.

- Outdoor temperature near the indoor temperature, so that temperature-driven (stack effect) infiltration will be small compared to the rate of mechanical ventilation being tested.

- Weather conditions as steady as possible, rather than changing. Overnight testing, or testing during a steady light rain, may be favorable in this regard.

Following the test, an engineering judgment is made about whether the test conditions were sufficiently steady-state to permit the age-of-air analysis (see criteria in Section 7.3 and examples in Section 8).

The operational details of the test protocol are described in Section 7. 


\subsection{Illustrative Cases}

To illustrate how our adapted age-of-air analysis applies in several airflow scenarios, we present here a series of five hypothetical case studies. These examples are useful as reference cases for interpreting field test results, as described in Section 8. For each analytical example, we perform the following steps:

1. Specify all the airflows for the hypothetical scenario.

2. Simulate field test data by calculating the tracer gas decay curves.

3. Analyze the decay curves with Equation 1.

4. Discuss the relationship between the airflow scenario and the results.

In each case, we make the following assumptions:

- The air in each room is fully mixed.

- All airflow rates are constant.

- Only two rooms are involved in the air exchange. In a home, more complex airflows may occur among more than two rooms. However, these examples illustrate important effects for analysis purposes. Actual field test results are shown in Section 8.

To simulate tracer-decay field tests, we analyze a general case that encompasses all five analytical examples. As shown in Figure 3, this analysis allows for airflow from Room A to Room B, but not from Room B to Room A. ${ }^{8}$ Each of the analytical examples that follow is a special case of this general case. This analysis is for illustration purposes only and is not part of the test and analysis protocol.

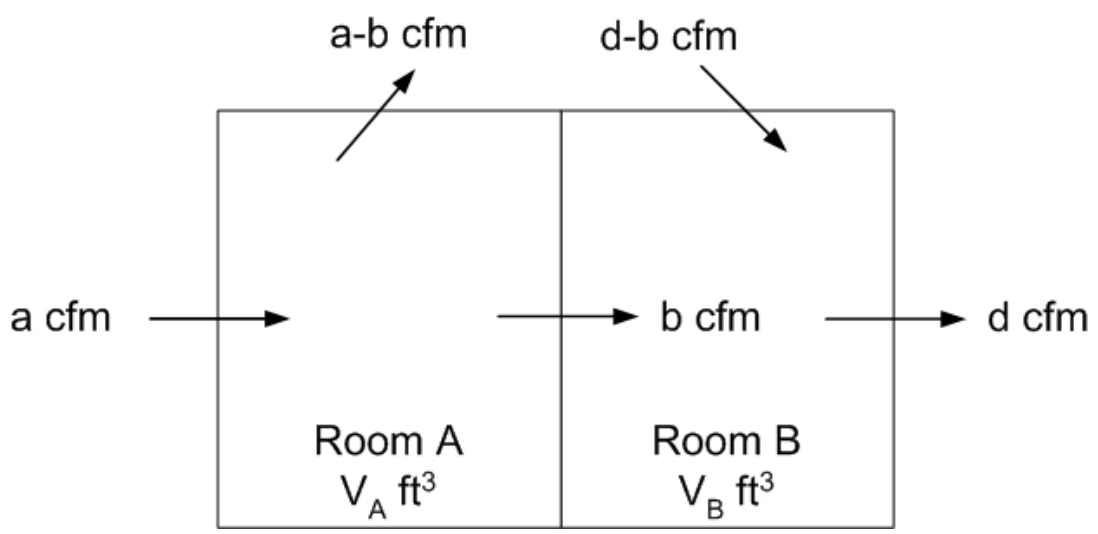

Figure 3. General case for Analytical Examples 1 to 5

In each room, the concentration is defined as (see Nomenclature section):

\footnotetext{
${ }^{8}$ In reality, two-way airflow may occur between rooms. However, such cases complicate the analysis and are not necessary for the purpose of these examples.
} 
$c=\frac{m}{V}$

In Room A, the tracer mass continuity equation is:

$a(0)-b\left(c_{A}\right)-(a-b) c_{A}=\frac{d m_{A}}{d t}$

This sets up the first-order, linear differential equation:

$\frac{d c_{A}}{d t}+\frac{a}{V_{A}} c_{A}=0$

which has the simple exponential (i.e., a single exponential function) solution:

$\frac{c_{A}}{c_{o}}=\exp \left(\frac{-a}{V_{A}} t\right)$

In Room B, the tracer mass continuity equation is:

$b c_{A}+(d-b)(0)-d c_{B}=\frac{d m_{B}}{d t}$

This sets up the first-order, linear differential equation, coupled to Equation 4:

$\frac{d c_{B}}{d t}-\frac{b}{V_{B}} c_{A}+\frac{d}{V_{B}} c_{B}=0$

Equation 7 has the compound exponential (i.e., multiple exponential functions with different decay rates) solution:

$\frac{c_{B}}{c_{o}}=p \cdot \exp \left(\frac{-a}{V_{A}} t\right)+q \cdot \exp \left(\frac{-d}{V_{B}} t\right)$

where:

$p=\frac{b V_{A}}{d V_{A}-a V_{B}}$

$q=1-p$

provided (as is true for all the cases considered): 
$d V_{A} \neq a V_{B}$

The specific analytical examples are described below and illustrated in Figures 4 to 9.

\subsubsection{Analytical Example 1: Single Room}

Airflow specifications. Figure 4 a shows the case of a single well-mixed room.

Intuition. Because this is a well-mixed enclosure, the common calculation of ACR applies.

$$
A C R=\frac{\text { Airflow }}{\text { Volume }}=\frac{10 \mathrm{ft}^{3} / \mathrm{min}}{1200 \mathrm{ft}^{3}} \cdot \frac{60 \mathrm{~min}}{\mathrm{hr}}=0.50 \mathrm{ACH}
$$

Field test simulation. With reference to Figures 3 and $4 \mathrm{a}$, the parameters for this case are:

$$
\begin{array}{ll}
\mathrm{V}_{\mathrm{A}} & =1200 \mathrm{ft}^{3} \\
\mathrm{~V}_{\mathrm{B}} & \mathrm{n} / \mathrm{a} \\
\mathrm{a} & =10 \mathrm{cfm} \\
\mathrm{b} & =10 \mathrm{cfm} \\
\mathrm{d} & \mathrm{n} / \mathrm{a}
\end{array}
$$

Thus, the tracer decay curve, based on Equation 5, is:

$$
\frac{c_{A}}{c_{o}}=\exp (-0.5 \cdot T)
$$

This curve, shown in Figure 4b, is a simple exponential function, as is always the result in a single well-mixed zone with steady airflow. The semilog plot of the tracer decay curve, shown in Figure $4 \mathrm{c}$, is a straight line, which corresponds to the simple exponential decay function.

Age-of-air analysis: From Equations 1 and 10:

$$
\tau=\frac{1}{c_{o}} \int_{0}^{\infty} c_{o} \exp (-0.5 \cdot T) d T
$$

This has the solution $\tau=2 \mathrm{hr}$, so

$$
R A o A=\frac{1}{\tau}=0.50 / h r
$$

Comment: For the single, well-mixed enclosure, the RAoA is the same as the ACR calculated in Equation 9. 


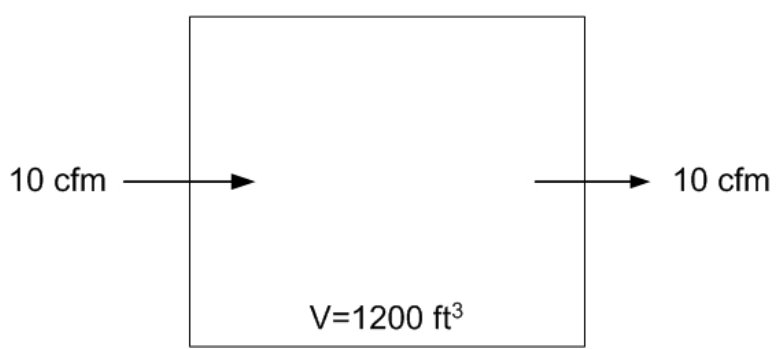

(a) Specified airflows

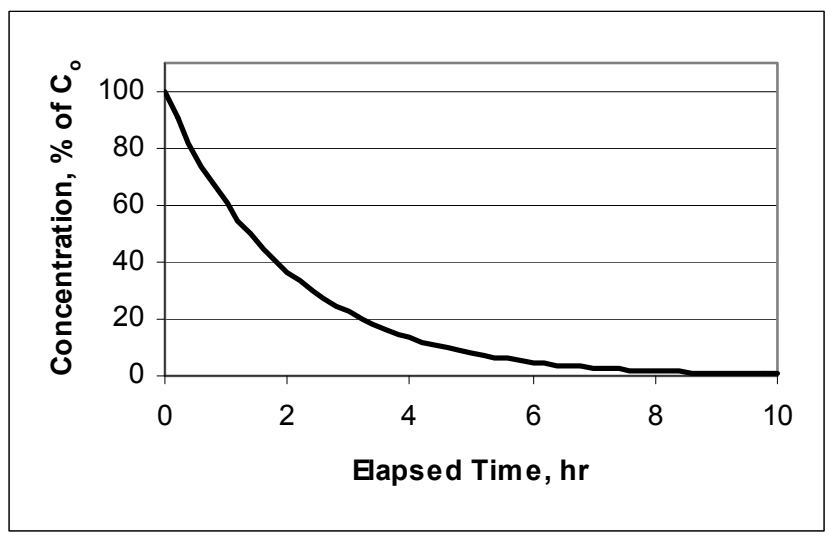

(b) Tracer decay curve in normal (arithmetic) format

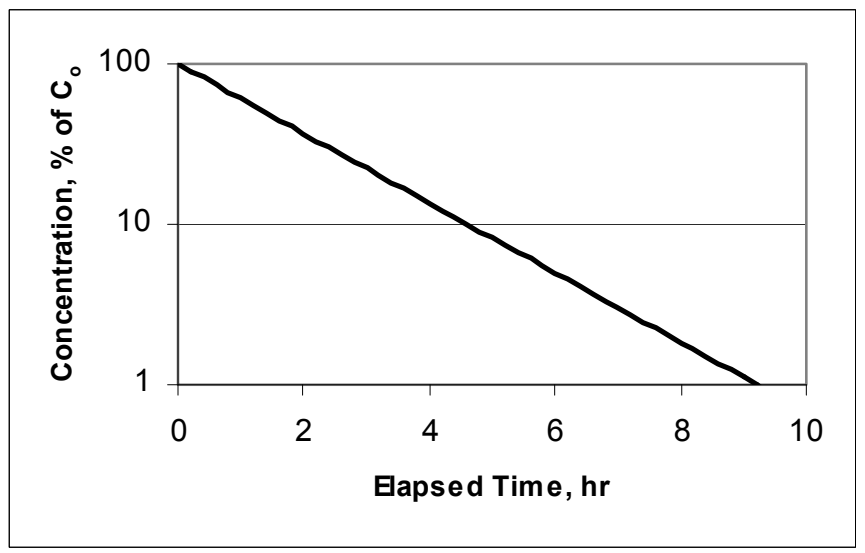

(c) Tracer decay curve in semilog format

Figure 4. Analytical Example 1: Single Room.

The decay curve is a simple exponential function. The semilog curve is linear. $\mathrm{RAOA}=\mathrm{ACR}=0.50 / \mathrm{hr}$ 


\subsubsection{Analytical Example 2: Two Independent Rooms}

Airflow specifications. Figure 5a shows the case of two independent rooms with no airflow between them.

Intuition. Each room is a well-mixed enclosure receiving outside air only (no interzonal airflow) with its own ACR. In Room A,

$$
A C R=\frac{\text { Airflow }}{\text { Volume }}=\frac{10 \mathrm{ft}^{3} / \mathrm{min}}{1000 \mathrm{ft}^{3}} \cdot \frac{60 \mathrm{~min}}{\mathrm{hr}}=0.60 \mathrm{ach}
$$

In Room B,

$$
A C R=\frac{\text { Airflow }}{\text { Volume }}=\frac{10 \mathrm{ft}^{3} / \mathrm{min}}{1500 \mathrm{ft}^{3}} \cdot \frac{60 \mathrm{~min}}{\mathrm{hr}}=0.40 \mathrm{ach}
$$

Field test simulation. With reference to Figures 3 and 5a, the parameters for this case are:

$$
\begin{aligned}
\mathrm{V}_{\mathrm{A}} & =1000 \mathrm{ft}^{3} \\
\mathrm{~V}_{\mathrm{B}} & =1500 \mathrm{ft}^{3} \\
\mathrm{a} & =10 \mathrm{cfm} \\
\mathrm{b} & =0 \mathrm{cfm} \\
\mathrm{d} & =10 \mathrm{cfm}
\end{aligned}
$$

From Equation 5:

$$
\frac{c_{A}}{c_{o}}=\exp (-0.6 \cdot T)
$$

From Equation 8:

$$
\begin{aligned}
& p=0 \\
& q=1
\end{aligned}
$$

$$
\frac{c_{B}}{c_{o}}=\exp (-0.4 \cdot T)
$$

In Figure 5b, both curves are simple exponential functions, with different decay rates. In Figure 5c, the semilog curves are both linear, with different slopes.

Age-of-air analysis. From Equations 1, 15, and 16, similarly to Analytical Example 1, the RAoA in Room A is $0.60 / \mathrm{hr}$ and in Room B is $0.40 / \mathrm{hr}$, matching the values of ACR found in Equations 13 and 14 . 


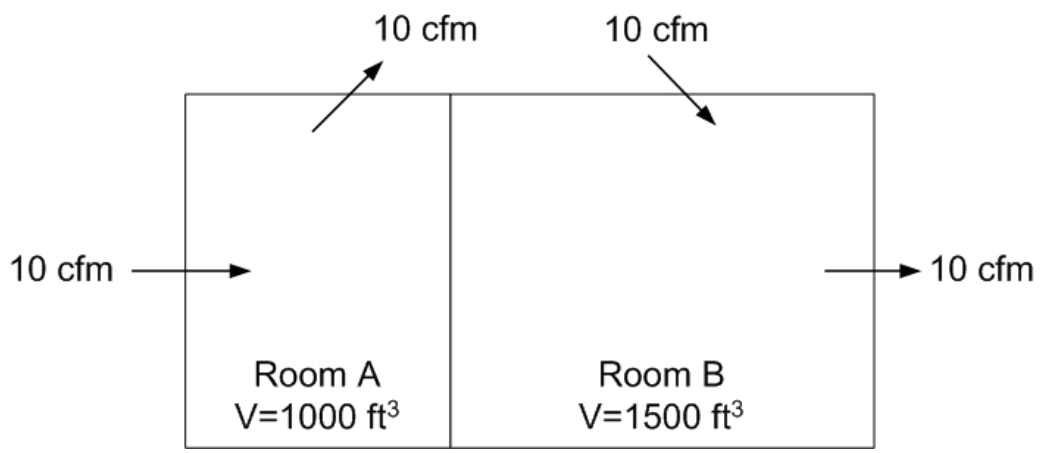

(a) Specified airflows

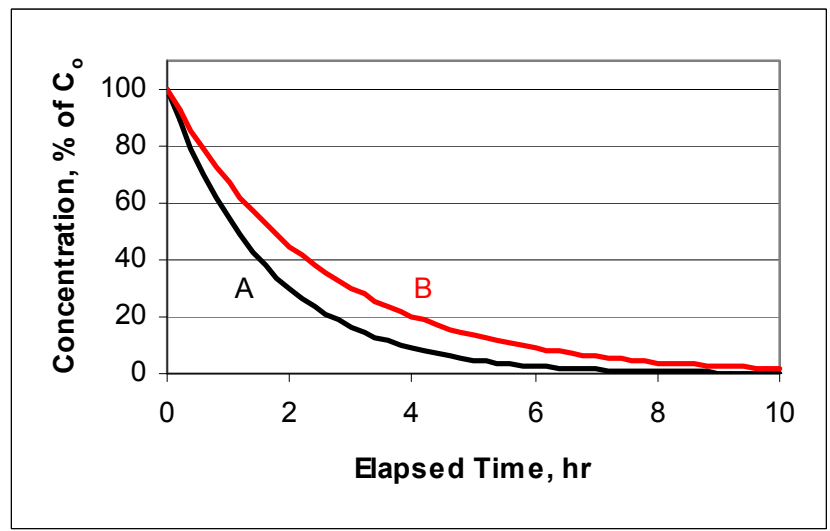

(b) Tracer decay curves in normal (arithmetic) format

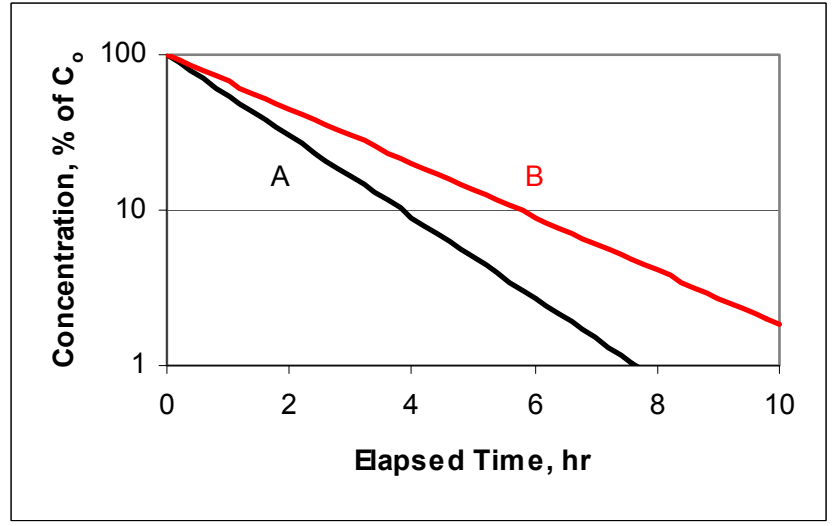

(c) Tracer decay curves in semilog format

Figure 5. Analytical Example 2: Two Independent Rooms.

Both decay curves are simple exponential functions. Both semilog curves are linear. RAOA = ACR = 0.60/hr in Room A and 0.40/hr in Room B. 
Comment. The purpose of including this example is to illustrate the appearance of the tracer decay curves, for comparison with the examples that follow.

\subsubsection{Analytical Example 3: Airflow in Series, Room A Smaller}

Airflow specifications. Figure 6a shows two rooms with airflow in series. That is, all of the airflow entering Room B comes from Room A.

Intuition. The air in Room A is likely to be fresher (less polluted) than the air in Room B, because Room A receives direct outside air while Room $B$ receives air that has passed through Room $A$ and may arrive polluted. The age-of-air is greater in Room B (thus the RAoA is smaller) because it includes time spent in both rooms.

Field test simulation. With reference to Figures 3 and $6 a$, the parameters for this case are:

$$
\begin{aligned}
\mathrm{V}_{\mathrm{A}} & =1000 \mathrm{ft}^{3} \\
\mathrm{~V}_{\mathrm{B}} & =1500 \mathrm{ft}^{3} \\
\mathrm{a} & =10 \mathrm{cfm} \\
\mathrm{b} & =10 \mathrm{cfm} \\
\mathrm{d} & =10 \mathrm{cfm}
\end{aligned}
$$

From Equation 5:

$$
\frac{c_{A}}{c_{o}}=\exp (-0.6 \cdot T)
$$

From Equation 8:

$$
\begin{aligned}
& \mathrm{p}=-2 \\
& \mathrm{q}=3 \\
& \frac{c_{B}}{c_{o}}=-2 \cdot \exp (-0.6 \cdot T)+3 \cdot \exp (-0.4 \cdot T)
\end{aligned}
$$

The Room A decay curve (Equation 17) is a simple exponential, but the Room B decay curve (Equation 18) is a compound exponential, involving two different decay rates. In Figure 6b, curve B looks different from the simple exponential shape of curve A. The difference may be described as an S-shape, or as an initial delay in the tracer gas decay. This delay occurs because the air entering Room B comes from Room A, carrying with it tracer gas. Room B has to wait for Room A to decay until it begins to decay. In Figure $6 \mathrm{c}$, the semilog plot for Room B is nonlinear, which is another way of recognizing the compound exponential behavior.

Age-of-air analysis. The RAoA in Room A, from Equation 17 (similarly to Analytical Example 1), is $0.60 / \mathrm{hr}$. In Room B, from Equations 1 and 18: 


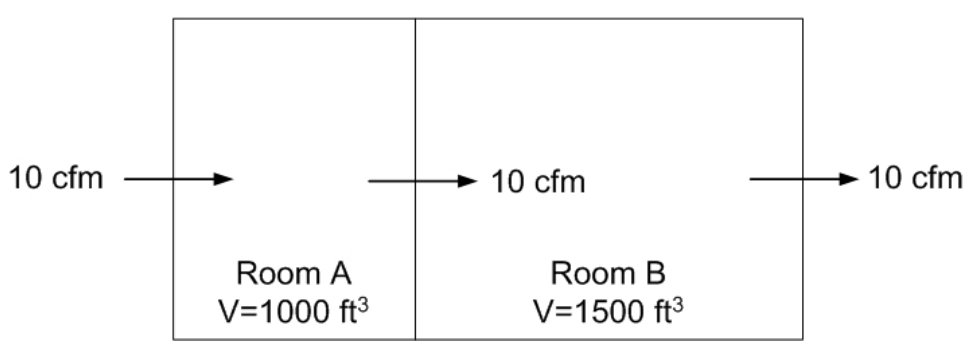

(a) Specified airflows

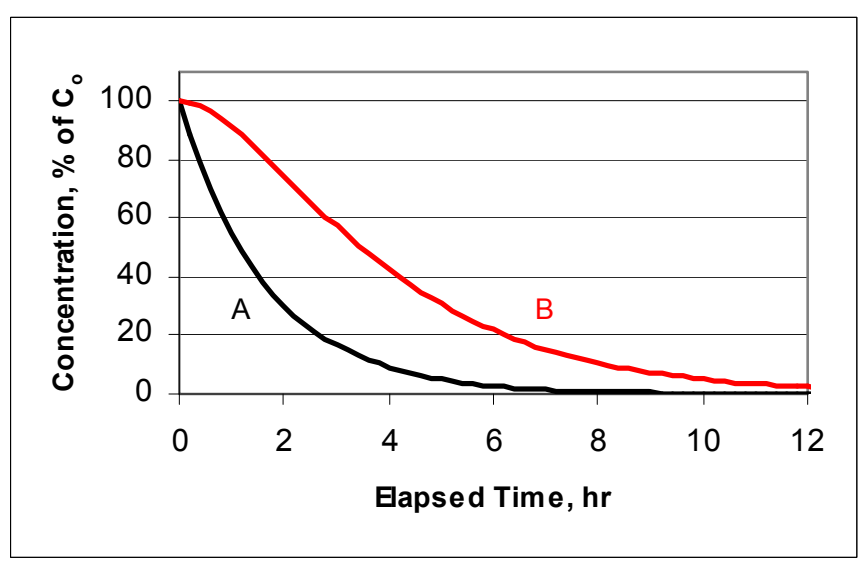

(b) Tracer decay curves in normal (arithmetic) format

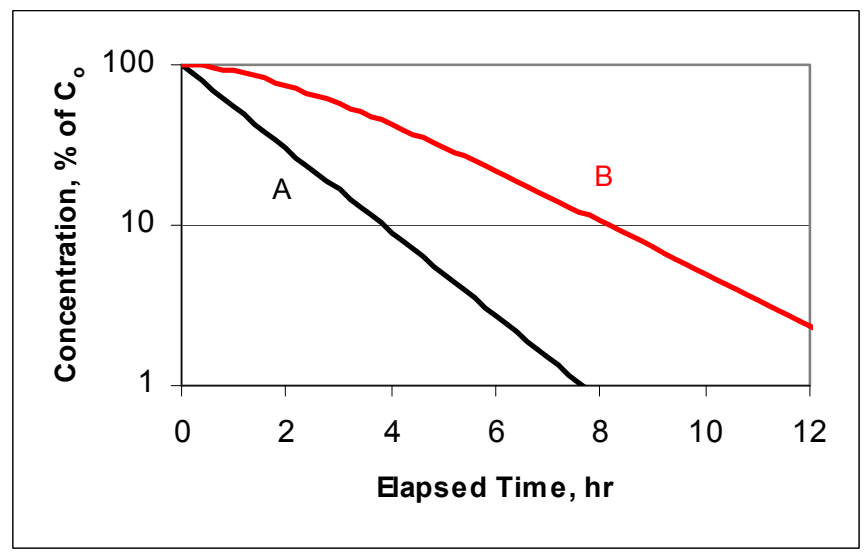

(c) Tracer decay curves in semilog format

Figure 6. Analytical Example 3: Airflow in Series, Room A Smaller.

The Room $B$ decay curve is a compound exponential function; the semilog curve is nonlinear. RAoA $=0.60 / \mathrm{hr}$ in Room A and $0.24 / \mathrm{hr}$ in Room $B$. 
$\tau=\frac{1}{c_{o}} \int_{0}^{\infty} c_{o}[-2 \cdot \exp (-0.6 \cdot T)+3 \cdot \exp (-0.4 \cdot T)] d T$

Thus $\tau=4.17 \mathrm{hr}$ and $\mathrm{RAoA}=0.24 / \mathrm{hr}$, significantly less than that in Room A, in agreement with our earlier intuition.

Comment. Toward the end of the time interval plotted in Figures $6 \mathrm{~b}$ and $6 \mathrm{c}$, after about 8 hours, curve B behaves similarly to a simple exponential, and its semilog curve looks linear in this region. This is because the effect of the faster decay rate $(0.6 / \mathrm{hr})$ becomes insignificant before that of the slower decay rate $(0.4 / \mathrm{hr})$. However, neither decay rate indicates the age-of air in Room B, which is represented by the area under the decay curve, including the influence of the initial delay.

\subsubsection{Analytical Example 4: Airflow in Series, Room A Larger}

This example, shown in Figure 7, is like the previous example except that the room volumes are switched, so the larger room is upstream in the airflow. The parameters and decay functions for this case are:

$$
\begin{array}{ll}
\mathrm{V}_{\mathrm{A}} & =1500 \mathrm{ft}^{3} \\
\mathrm{~V}_{\mathrm{B}} \quad=1000 \mathrm{ft}^{3} & =10 \mathrm{cfm} \\
\mathrm{a} \quad=10 \mathrm{cfm} \\
\mathrm{b} \quad=10 \mathrm{cfm} \\
\mathrm{d} \quad \\
\frac{c_{A}}{c_{o}}=\exp (-0.4 \cdot T) \\
\mathrm{p}=3 \\
\mathrm{q}=-2 \\
\frac{c_{B}}{c_{o}}=3 \cdot \exp (-0.4 \cdot T)-2 \cdot \exp (-0.6 \cdot T)
\end{array}
$$

Compared to Analytical Example 3, Room A now has a slower decay rate and a smaller value of RAoA: 0.4/hr. Equation 21 is the same as Equation 18 with the order of the terms reversed. Thus, Room $B$ again registers $\tau=4.17 \mathrm{hr}$ and $\mathrm{RAoA}=0.24 / \mathrm{hr}$. Having spent more time in Room A, but less time in Room B, the age-of-the air in Room B is the same as in the previous example.

Insight may be gained by comparing the curves in Figure 7 to those in Figure 6 . In Figure 7, the two curves have the same decay rate toward the end of the interval (the same slope in Figure $7 \mathrm{c}$ ). This is shown by Equation 21, in which the slower decay rate $(0.4 / \mathrm{hr})$ matches that of Equation 20. This does not mean the two rooms experience equivalent air exchange. Although the tracer decay test measures a transient system response, the age-of-air is a steady-state phenomenon, related to the route taken by the steady airflow through the two rooms. The age-of-air calculation is based on the area under the decay curve, which includes the influence of the time delay in the decay of the tracer gas. This time delay corresponds to the route taken by the airflow. 


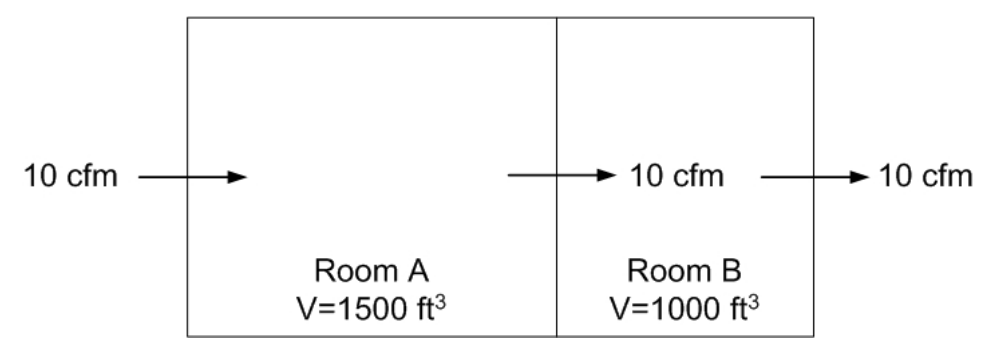

(a) Specified airflows

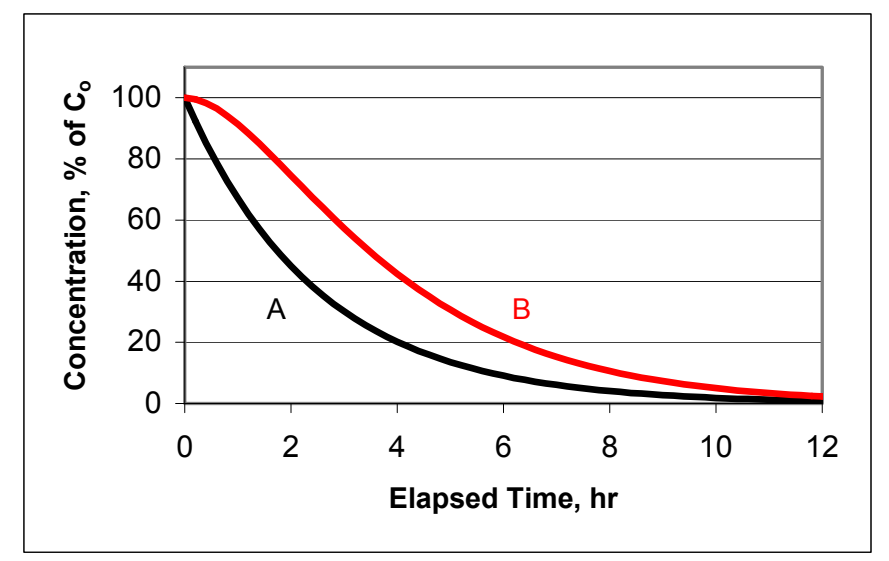

(b) Tracer decay curves in normal (arithmetic) format

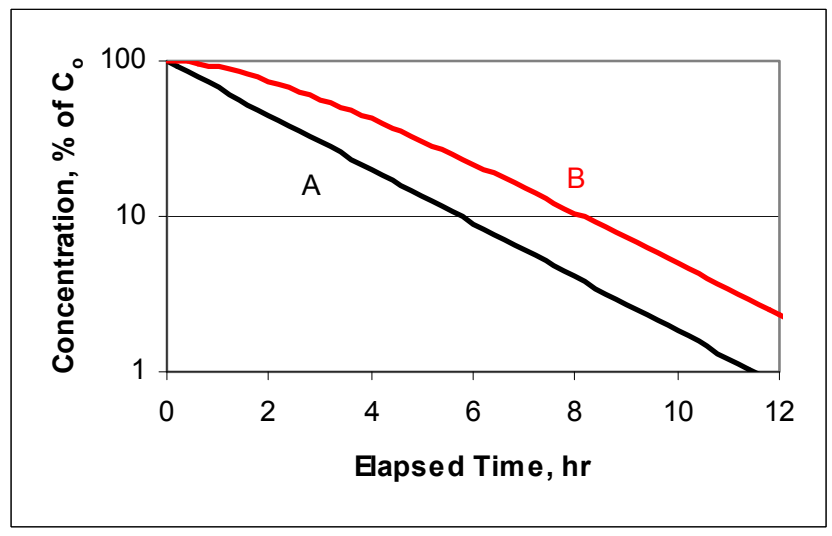

(c) Tracer decay curves in semilog format

Figure 7. Analytical Example 4: Airflow in Series, Room A Larger.

The Room $B$ decay curve is a compound exponential function; the semilog curve is nonlinear. Decay rates (slopes of semilog curves) are equal near the end of the time interval. $\mathrm{RAOA}=0.40 / \mathrm{hr}$ in Room A and $0.24 / \mathrm{hr}$ in Room B. 
The fact that the air is coming from another room makes it older, and likely to contain more pollutants. The tracer decay test captures this effect at the beginning of the test interval. Trying to evaluate the test results by looking only at the decay rates at the end of the interval can be misleading.

To further illustrate this point, Figure 8 shows a calculation of apparent instantaneous decay rate for this example. This calculation is based on the slope of the decay curve, interpreted as a simple exponential function:

$$
c=c_{o} \exp (-\kappa T)
$$

$\frac{d c}{d T}=-\kappa \cdot c_{o} \exp (-\kappa T)$

$\kappa=\frac{-1}{c} \frac{d c}{d T}$

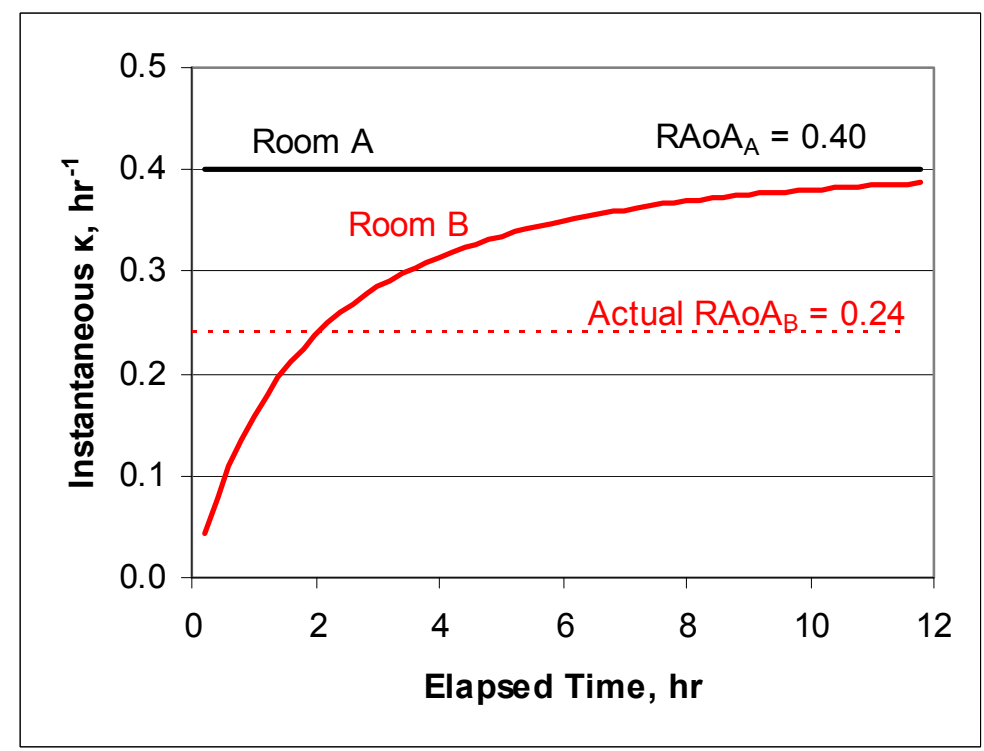

Figure 8. Apparent instantaneous decay rates for Analytical Example 4

Our gas analyzer software performs this calculation routinely. As shown in Figure 8, the result for Room A is correct, because the tracer decay curve is indeed a simple exponential function. For Room B, the instantaneous decay rate appears to vary with time and approaches curve A at the end of the interval. However, this is an erroneous interpretation. The age-of-air in Room B is actually a constant, which is captured by the area under the transient decay curve (shown by the dotted line in Figure 8). The instantaneous decay rate does not have the same meaning here as it does in a single well-mixed zone with a simple exponential decay curve. 


\subsubsection{Analytical Example 5: Partial Series Airflow}

Airflow specifications. The final analytical example, shown in Figure 9, involves the same airflow pattern that was used in the cigar-smoker illustration in Section 3 (Figures 1 and 2). Room B receives some direct outside air $(4 \mathrm{cfm})$ as well as some interzonal airflow from Room A $(6 \mathrm{cfm})$.

Intuition. Based on our discussion in Section 3, the air in Room A is likely to be fresher (less polluted) than the air in Room B. By comparison to the other analytical examples, Room B seems better ventilated here than in Example 3, but not as well as in Example 2.

Field test simulation. With reference to Figures 3 and $9 \mathrm{a}$, the parameters for this case are:

$$
\begin{aligned}
\mathrm{V}_{\mathrm{A}} & =1000 \mathrm{ft}^{3} \\
\mathrm{~V}_{\mathrm{B}} & =1200 \mathrm{ft}^{3} \\
\mathrm{a} & =10 \mathrm{cfm} \\
\mathrm{b} & =6 \mathrm{cfm} \\
\mathrm{d} & =10 \mathrm{cfm}
\end{aligned}
$$

From Equation 5:

$$
\frac{c_{A}}{c_{o}}=\exp (-0.6 \cdot T)
$$

From Equation 8:

$\mathrm{p}=-3$

$\mathrm{q}=4$

$$
\frac{c_{B}}{c_{o}}=-3 \cdot \exp (-0.6 \cdot T)+4 \cdot \exp (-0.5 \cdot T)
$$

The decay curve for Room B is a compound exponential, although this fact is less obvious in Figure $9 b$ than in Figures $6 b$ and $7 b$. However, in Figure 9c, the Room B semilog plot shows up as nonlinear, indicating its difference from a simple exponential. This curve is more typical of a tracer gas decay curve measured in a field test in a home. It would be a mistake to assume the curve is a simple exponential for analytical purposes. The field test must be continued until the curve resembles a simple exponential, which is best determined by observing the semilog curve. In Figure 9c, the Room B semilog curve appears to be linear after about 8 hours of elapsed time. This time varies from case to case, depending on the house geometry and the airflows affecting the room. 


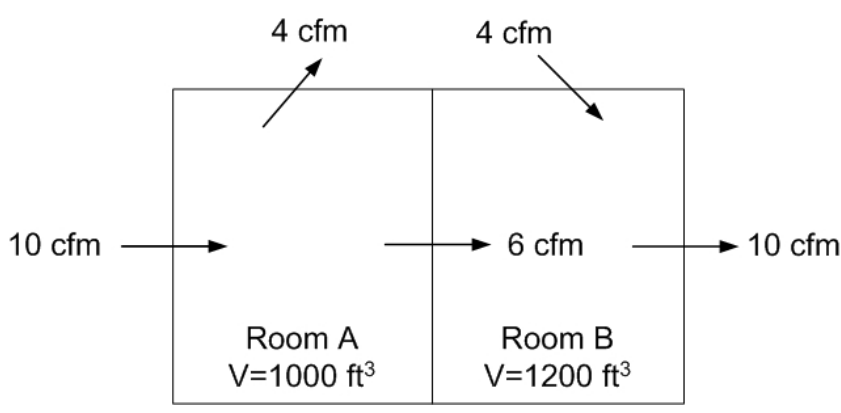

(a) Specified airflows

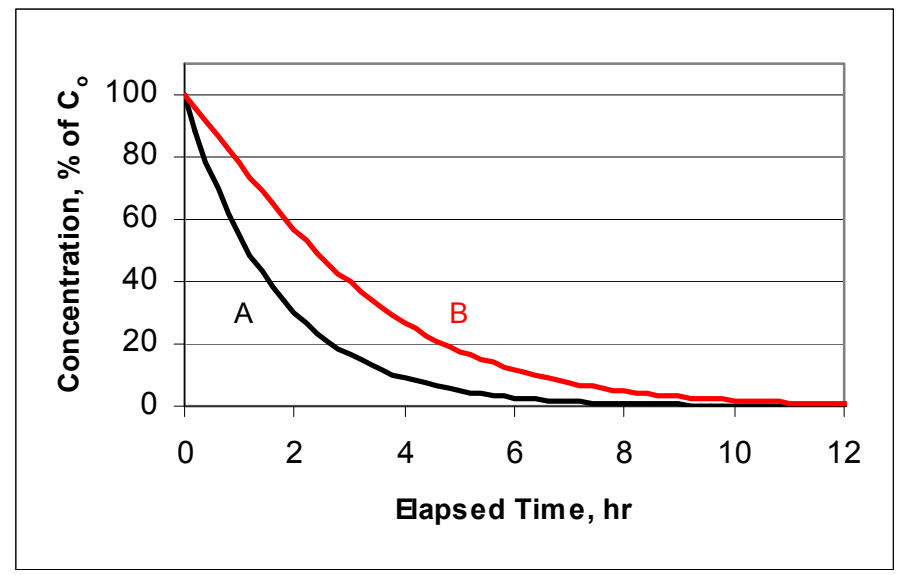

(b) Tracer decay curves in normal (arithmetic) format

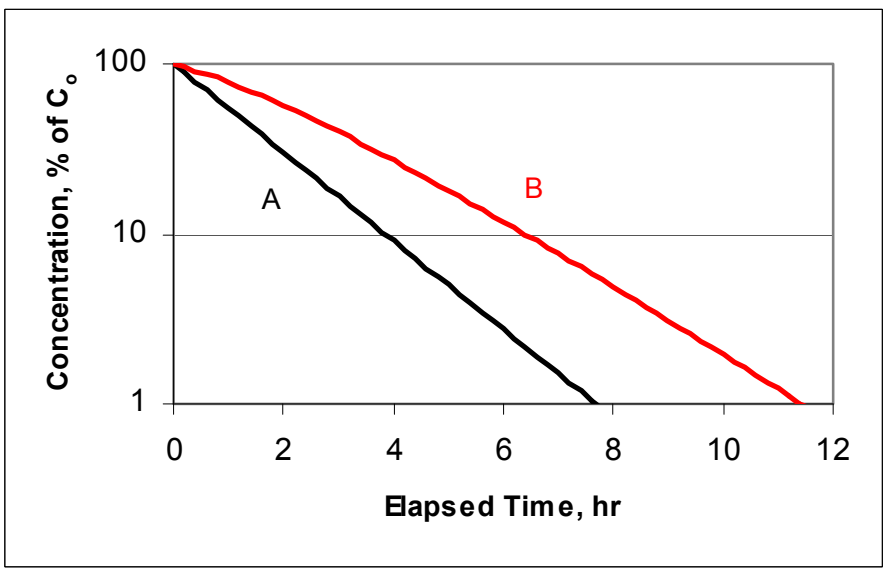

(c) Tracer decay curves in semilog format

Figure 9. Analytical Example 5: Partial Series Airflow. The Room B decay curve is a compound exponential, although not as conspicuously as in the previous two examples. RAOA = 0.60/hr in Room A, 0.33/hr in Room B. 
Age-of-air analysis. In Room A, the RAoA is again the same as the ACR: 0.60/hr. In Room B,

$\tau=\frac{1}{c_{o}} \int_{0}^{\infty} c_{o}[-3 \cdot \exp (-0.6 \cdot T)+4 \cdot \exp (-0.5 \cdot T)] d T=3 h r$

$\mathrm{RAoA}=1 / 3=0.33 / \mathrm{hr}$

Comment. The RAoA value of $0.33 / \mathrm{hr}$ is between the values from Analytical Example $3(0.24 / \mathrm{hr})$ and Analytical Example $2(0.40 / \mathrm{hr})$, in agreement with our intuition. 


\section{The Graph Overlay Method}

Age-of-air analysis, as described in Section 5, requires sufficiently steady conditions during the test period. Because of this requirement, we often try to schedule field tests during mild weather seasons to minimize thermally driven infiltration, so its magnitude will be small compared to more constant mechanical ventilation rates. In addition, we hope for calm weather during the test period, so that wind, which also drives infiltration and tends to fluctuate rapidly, will be minimal.

However, field tests must usually be scheduled weeks or months in advance to coordinate access to the home when construction is sufficiently complete and before the home is occupied, as well as the availability of the test engineer and the necessary equipment. An engineering judgment of whether the actual test conditions were sufficiently steady state for age-of-air analysis cannot be made until after the tracer decay data are recorded. Thus, we are sometimes disappointed in our inability to proceed with the age-of-air analysis. In these cases, we can use an alternative, less precise, analysis method.

The Graph Overlay Method is applied by superimposing a family of calculated reference curves, based on simple exponential decay, over the actual decay curves measured in the field test. The calculated curves correspond to a gradation of ACRs in a hypothetical single-zone scenario. Figure 10 shows a sample reference grid. If a measured decay curve lies entirely above or below one of the calculated curves, corresponding to, say, $\mathrm{X} \mathrm{ACH}$, we can describe the RAoA as being less than or greater than $\mathrm{X}$, respectively. (Note the reverse relationship between curve position and ACR or RAoA.) Similarly, if a measured decay curve lies entirely between two of the calculated decay curves, corresponding to, say, $\mathrm{X}$ and $\mathrm{Y} \mathrm{ACH}$, we can describe the RAoA as being in the range $\mathrm{X}$ to $\mathrm{Y}$. The following formula is used to generate the reference curves:

$$
\frac{c}{c_{o}}=\exp (-A C R \cdot T)
$$

Examples demonstrating the use of this method are shown in Section 8 (Field Examples 3 and 4). 


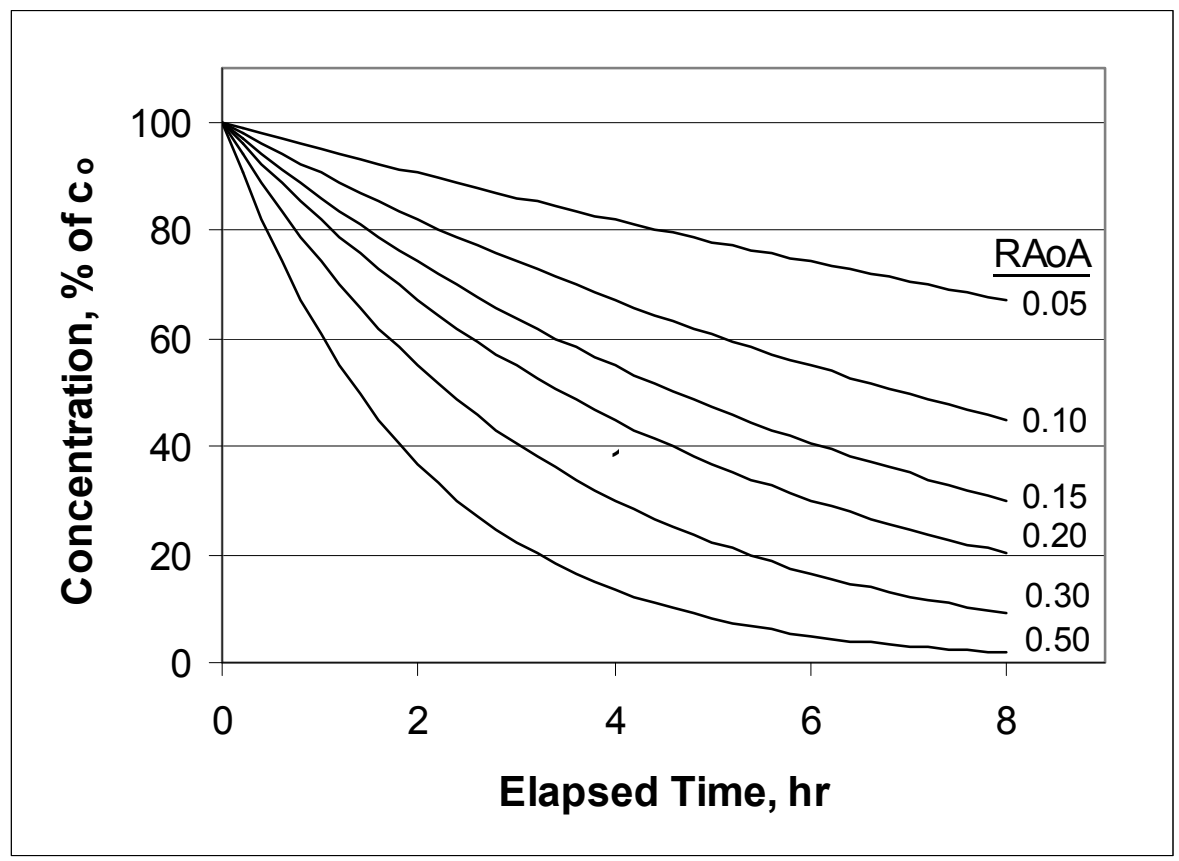

Figure 10. Grid for use with the Graph Overlay Method 


\section{Test and Analysis Protocol}

The test and analysis protocol consists of three phases:

- Planning the field test.

- Performing the test.

- Analyzing the data.

Each of these phases is described below.

\subsection{Planning the Field Test}

The first step in planning is to identify the goals of the field test, i.e., the study questions to be answered. Examples of field test goals include:

- Evaluate a ventilation system based on room-to-room distribution of outside air.

- Compare two mechanical ventilation systems based on room-to-room distribution of outside air.

- Compare the performance of a mechanical ventilation system with room doors open versus closed.

The second step is to identify the specific test cases that will provide answers to the study questions. Examples of test cases include:

- Mechanical ventilation system \#1 running, room doors closed.

- Mechanical ventilation system \#1 running, room doors opened.

- Mechanical ventilation system \#2 running, room doors closed.

- Mechanical ventilation system \#2 running, room doors opened.

As part of Step 2, designate the locations in the home (sample points) where tracer gas concentration will be measured. (See Section 7.2.2 for further details.)

The third planning step is to estimate the time needed to conduct the series of tests. For age-of-air analysis, each tracer decay test should be continued for about $1 \frac{1}{2}$ air changes in the least-ventilated portion of the home. For example, if a ventilation system is designed for a whole-house ACR of $0.20 \mathrm{ACH}$, each air change will take $1 / 0.20=5 \mathrm{hr}$, so $1 \frac{1}{2}$ air changes will take 7.5 hours. If one or more zones receive significantly less outside air, more time will be needed. For a typical series of tests planned to evaluate several ventilation configurations, a test period of several days is needed.

Some guidelines to consider when planning a field test are as follows: 
1. Try to plan for mild weather, including low wind speeds and moderate outdoor temperature. Reasons for this include:

- Less infiltration and less fluctuation in infiltration help to achieve the steady conditions needed for age-of-air analysis.

- Low infiltration improves our ability to compare performance in a sequence of tests, because we can attribute differences to changes in the ventilation mode under test rather than changes in the weather.

- Testing during mild weather checks the ability of the mechanical ventilation system to provide adequate ventilation with minimal assistance from weather-driven infiltration. Because most climates have periods of mild weather at certain times of the year, ventilation performance at these times is a significant concern.

2. Try to schedule the test when construction of the home will be sufficiently completed so that the ventilation system performance will correspond to the finished building.

3. Schedule the test when the home will be unoccupied, to avoid disrupting occupants and interference with the test conditions.

4. Review the procedures in Section 7.2 and verify that enough time is scheduled to complete the tests.

5. If the home includes an unconditioned basement, review the concerns discussed in Section 7.4 .

6. Coordinate the test schedule with the availability of the test engineer and the test equipment.

\section{2 $\quad$ Performing the Test}

The test and analysis procedure is described below. Refer to the user manuals for the various pieces of test equipment for further instructions.

\subsubsection{Equipment}

- Gas analyzer. We use a Brüel and Kjær model 1302 multi-gas monitor (Brüel and Kjær 2007).

- Multi-point sampler. We use Brüel and Kjær model 1303 (Brüel and Kjær 2007).

- Tubing for gas sampling, compatible with the multi-point sampler and the tracer gas. We use 2.5-mm ID polyethylene tubing.

- Wooden stands to position the sampling points near the center of each room. We use a height of 4 feet above the floor, which is half the height of an 8-foot ceiling.

- Tracer gas. We use $\mathrm{SF}_{6}$. We use a 0.5-lb lecture bottle, which holds enough gas for about 10 to 20 tests. Using a small bottle minimizes the asphyxiation hazard in case it leaks in a confined space.

- Room mixing fans. We use 12-inch box fans, 8-inch oscillating fans, or ceiling fans if present in the home, on low speed in each case. 
- Interzonal mixing fans. We recommend the use of the air handler fan for this purpose, when one is present in the home. Otherwise, we use two, 200-cfm fans with 20-foot flex ducts attached; the flex ducts are typically deployed between levels in the home.

- Room heaters. We use a 1,500-W portable electric heater for each room. In homes with forced-air heat, we use these room heaters to maintain a uniform, normal temperature throughout the home without running the air handler for heating purposes. This enables us to test infiltration and ventilation modes independently of the heating system.

- Room temperature sensors and heater controllers. We use a Campbell Scientific, Inc. CR$10 \mathrm{X}$ datalogger for this purpose, with additional hardware as needed.

- Weather station. We use outdoor temperature and wind speed sensors with the CR-10X datalogger to monitor weather during the test, for use in interpreting the test data.

- Computer and software compatible with the gas analyzer and multi-point sampler to control the test equipment, record the data, and monitor the data during the test.

\subsubsection{Setup}

- Install the gas analyzer and multi-point sampler in the home to be tested.

- Deploy tubing from the multiplexer to each designated sample point. We recommend points near the center of each room at a height of 4 feet above the floor.

- Install the room mixing fans and room heaters in each room. Use the room air temperature to control the heater, and verify uniform and constant air temperature throughout the house.

- Program the sampler to draw a sample from each sequential location at the minimum acceptable sampling interval, which depends on the length of the tubing, the flow rate through the tubing, and the response time of the gas analyzer. We use an interval of about 2 minutes per location; thus, six locations may be sampled at an interval of about 12 minutes.

- Check and adjust the zero calibration of the gas analyzer before injecting any tracer gas.

\subsubsection{Preliminary Test}

Following the setup of the equipment, and before beginning the planned series of tests, we recommend a preliminary test in which multiple sample points are placed in one or more rooms to verify that good mixing is achieved within the rooms. This is especially important when the test engineer is becoming familiar with the procedure, the test equipment, or the test configuration, to verify that the room mixing fans are performing adequately. An example of such a test is described in Section 8, Field Example 1. However, if the planned series of tests includes multiple sample points within rooms, this preliminary test is not necessary.

\subsubsection{Testing}

- Turn on the measurement equipment and synchronize time codes where applicable.

- Turn on the room mixing fans and room heaters. Verify steady, uniform temperatures throughout the house. 
- Turn on the interzonal mixing fan(s). If practical, turn off the ventilation system. Of course, if the ventilation system is integral with the air handler that is used for mixing, this will remain on.

- Use a small, hand-held compressed gas cylinder to inject the tracer gas. Gas may be injected near the main air return with the air handler running, or injected into each room. Store the gas cylinder outside the house and away from any air intakes when injection is completed, or verify that the cylinder is not leaking, to avoid leakage from the cylinder into the house during the test.

- Verify an initially well-mixed condition, at the minimum concentration necessary for the test. This normally takes about one hour. We recommend a concentration of at least 200 times the lower detection limit of the instrument. This allows for a factor of 10 in tracer decay during the test, and a factor of 20 above the lower detection limit at the end of the test, for 5\% accuracy. For our gas analyzer, the lower detection limit is 10 PPB; 200 times 10 PPB is 2 PPM; we aim for 5 PPM as a margin above the minimum. Because $\mathrm{SF}_{6}$ has a 100-year global warming potential 22,200 times that of carbon dioxide per pound (IPCC 2001), we avoid using more than necessary. (The infrared absorptivity of $\mathrm{SF}_{6}$, which makes it a greenhouse gas, is the same property the gas analyzer uses to measure its concentration.)

- Designate a data sample time as Point A. Simultaneously start the ventilation mode to be tested and stop the interzonal air mixing, unless the ventilation system to be tested is integral with the air handler.

- Monitor the tracer gas concentration data throughout the duration of the test. Continue the measurements until the following conditions are met, noting this time as Point B:

$>$ Priority: Continue the test until the shape of each decay curve is a simple exponential, which appears as a straight line in a semilog plot, for a long enough time to estimate the decay constant (see analytical examples in Section 5 and field examples in Section 8). Age-of-air analysis can be conducted only if this condition is met.

Secondary: When practical, continue the test until the highest concentration among the measurement points is about $20 \%$ of the initial value, indicating that the equivalent of 1.5 air changes have occurred. Meeting this condition helps to reduce errors caused by extrapolating the decay curves to infinity for analysis purposes, because the area under the extrapolated curves is small compared to the area under the measured curves. However, if it is difficult to meet this condition, due to a change in weather conditions or exhaustion of the time available for the test, age-of-air analysis is still possible if the first condition (above) has been met.

\subsection{Analyzing the Data}

After completing the test procedure outlined above, review the tracer gas concentration data, and make an engineering judgment about the analysis method to be used. (Examples are provided in Section 8 to illustrate and discuss this decision.) The options are:

- If the test conditions are significantly unsteady, use the Graph Overlay Method described in Section 6 and illustrated in Section 8, Field Examples 3 and 4.

- If the test conditions are sufficiently steady and the decay curves are closely grouped, use the Air-Change Rate method described below and illustrated in Section 8, Field Example 2. 
- If the test conditions are sufficiently steady and the decay curves are not closely grouped, use the age-of-air method described below and illustrated in Section 8, Field Examples 1, 5 and 6.

\subsubsection{Air-Change Rate Method}

Verify that the following conditions are met:

- The tracer decay curves are so closely grouped that it is difficult to distinguish one from another.

- The decay curves appear linear in a semilog plot, over the span between Point A and Point B as identified in the test procedure (Section 7.2.4).

Calculate the ACR as:

$$
A C R=\frac{\ln \left(c_{A} / c_{B}\right)}{T_{B}-T_{A}}
$$

\subsubsection{Age-of-Air Method}

The infinite integral in Equation 1 (Section 5) is divided into two intervals, I and II. For the duration of the experiment (Interval I, between Points A and B described in Section 7.2.4), measured values of tracer gas concentration are integrated numerically, using the trapezoid rule. Then the residual portion of the decay curve (Interval II) is estimated by extrapolating the curve as a simple exponential function, which is matched to the decay rate of the final portion of the measured decay curve, and integrating analytically. Then

$$
\tau=\tau_{I}+\tau_{I I}
$$

Based on the trapezoid rule, calculate:

$$
\tau_{I}=\frac{\Delta T}{c_{A}}\left[\frac{1}{2}\left(c_{A}+c_{B}\right)+\sum_{i=i_{A}+1}^{i_{B}-1} c_{i}\right]
$$

Examine the semilog plot of the tracer decay curve, and identify the time (Point $C$ ) when the curve becomes linear. Point $\mathrm{C}$ should be selected as far from Point $\mathrm{B}$ (at the end of the test period) as possible, so the effect of any irregularities in the curve will be small compared to the measured concentration difference. However, Point $\mathrm{C}$ must be in the linear portion of the semilog curve so the portion of the curve between Point $\mathrm{C}$ and Point $\mathrm{B}$ is a good match to the assumed simple exponential function used for the extrapolation. Then the decay constant $\lambda$ is calculated based on the concentration over the interval from Point $\mathrm{C}$ to Point B:

$$
\lambda=\frac{\ln \left(c_{C} / c_{B}\right)}{T_{B}-T_{C}}
$$


The tail of the truncated curve is estimated with the function $c=c_{B} \cdot \exp \left(-\lambda T^{\prime}\right)$, where $T^{\prime}=T-T_{B}$. Then:

$$
\begin{aligned}
& \tau_{I I}=\frac{c_{B}}{c_{A} \lambda} \\
& \tau=\tau_{I}+\tau_{I I} \\
& R A o A=\frac{1}{\tau} .
\end{aligned}
$$

\subsection{Special Consideration for Unconditioned Basements}

Unconditioned basements present a special case for the test protocol for the following reasons:

- The temperature in an unconditioned basement is typically lower than that in the conditioned space.

- Air exchange between the basement and the conditioned space may be difficult to avoid, especially if an air handler or ductwork is present in the basement.

For our purposes here, the definition of an unconditioned basement is one in which the temperature is normally significantly different than that in the conditioned space, raising the concern described just above. This condition complicates the test protocol as follows:

- If we do not dose the basement with tracer gas, air exchanges during the test may affect the test results. Such an effect would be misleading, because the basement air would dilute the tracer gas in the conditioned space, although it does not represent actual outside air flow.

- If we heat the basement to the uniform house temperature, we change the system we are testing.

- If we include the basement in the tracer gas dosing and the preliminary whole-house mixing, we cannot avoid heating the basement.

Because of these concerns, we recommended the following procedure:

- Use room heaters to maintain a normal comfort temperature in the conditioned space, but leave the basement unheated.

- Place a room mixing fan and a tracer sampling point in the basement.

- Dose the conditioned space with tracer gas and mix well.

- Dose the basement separately.

- Monitor the tracer concentrations in the conditioned space and in the basement, and adjust the dosing until the two concentrations are equal.

- Stop the house mixing and begin the tracer decay test interval.

We have not yet attempted to use this procedure, so further experience is needed to evaluate its practicality. 


\section{Field Test Examples}

In this section, we present sample results from six actual field tests conducted using the protocol described in Section 7. Each example demonstrates a particular set of conditions that is typical in this type of testing. Engineering judgment is used in each case to choose the appropriate method of analysis: (1) the Graph Overlay Method, (2) calculation of ACR, or (3) room-by-room age-of-air analysis. An overview discussion of the test conditions related to steady and unsteady behavior is presented in Section 8.7.

\subsection{Field Example 1}

In most of our tests, we place each of the six sample points available with our test equipment in a different room to maximize coverage of the house. However, we recommend a preliminary test in which multiple sample points are placed in one or more rooms to verify that good mixing is achieved within the rooms. This is especially important when the test engineer is becoming familiar with the procedure, the test equipment, or the test configuration, to verify that the room mixing fans are performing adequately.

In March 2001 we conducted testing in the three-story, 1,700- $\mathrm{ft}^{2}$ home of NREL staff member Craig Christensen in Boulder, Colorado. During the test, the outdoor temperature ranged from $52^{\circ} \mathrm{F}$ to $58^{\circ} \mathrm{F}$, the wind speed ranged from 0.7 to $2.0 \mathrm{mph}$, and the indoor temperature ranged from $72^{\circ} \mathrm{F}$ to $79^{\circ} \mathrm{F}$ because of large solar gains. Each of the three levels in this home features an open architecture style, in which all the air can mix freely, with the exceptions of a den on the middle level, a laundry room on the top level, and $2 \frac{1}{2}$ bathrooms. During the tests, these room doors were kept open and small electric fans were used to mix the air on each level. Stairway doors were also kept open for this test. A heat recovery ventilator is ducted to supply and exhaust ports on each of the three levels, with a total airflow of about $80 \mathrm{cfm}$. Because there are only three distinct main rooms, we placed two sample points on each level, near the opposite ends (east and west) of the long dimension of the home. As shown in Figure 11a, the east and west decay curves on each level are almost indistinguishable from one another, while significant differences are shown among the three levels. This indicates that the mixing on each level is very good, because differences within each level are small compared to the differences among the levels, which are the object of measurement. On the semilog graph in Figure 11b, the decay curves for the upper and middle levels appear linear throughout the test period. The curves for the lower level appear linear after about 2.0 hours of elapsed time, so we designate this time as Point $\mathrm{C}$ in the age-of-air analysis protocol (Section 7.3.2). The results of the age-of-air analysis are shown in Table 1 and Figure 12. The lower level receives more outside air than the other two levels. This difference may be due to infiltration or an imbalance in the ducted airflow from the heat recovery ventilator to the ports on each level.

Table 1. RAoA results for Field Example 1

\begin{tabular}{|c|c|c|c|c|c|c|}
\hline Location & Lower East & Lower West & Middle East & Middle West & Upper East & Upper West \\
\hline RAoA, $\mathbf{h r}^{-1}$ & 0.580 & 0.587 & 0.387 & 0.389 & 0.345 & 0.343 \\
\hline
\end{tabular}




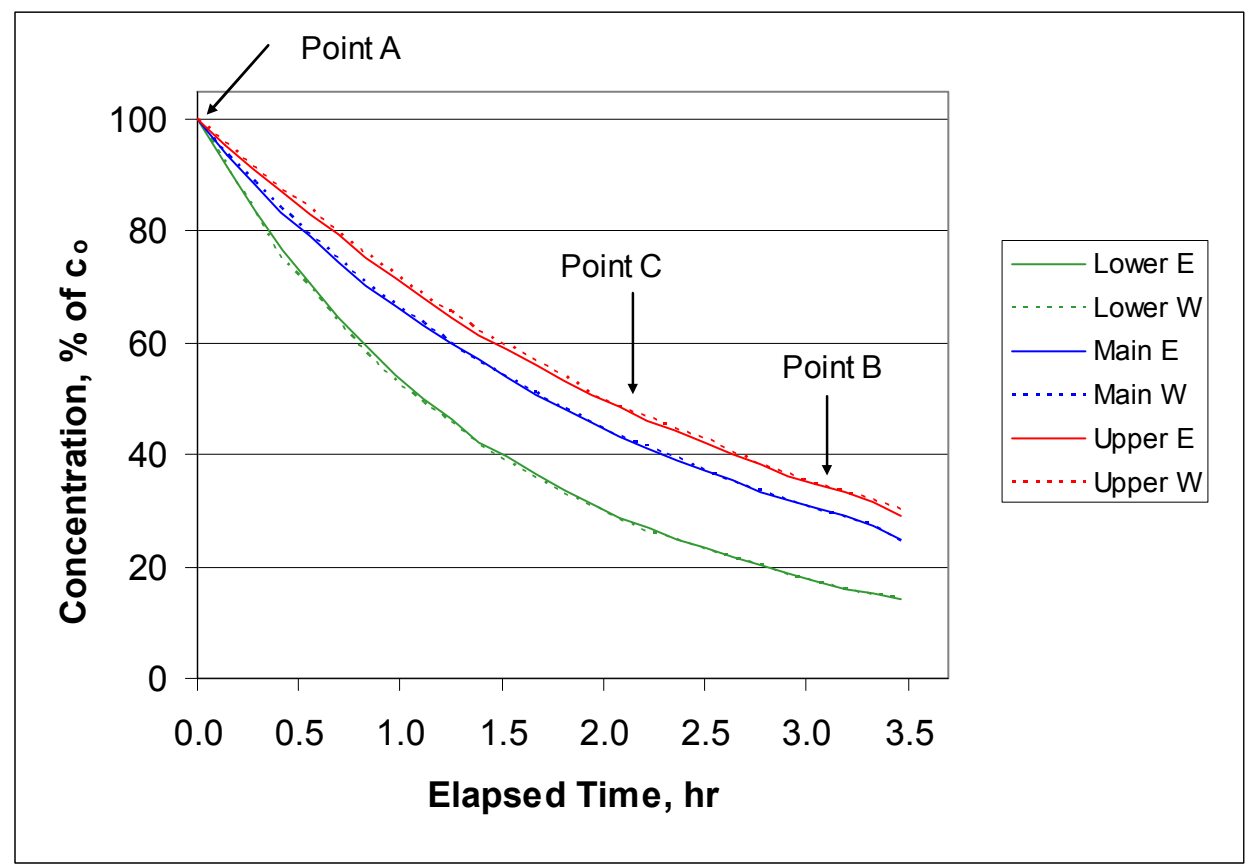

(a) Tracer decay curves in normal (arithmetic) format

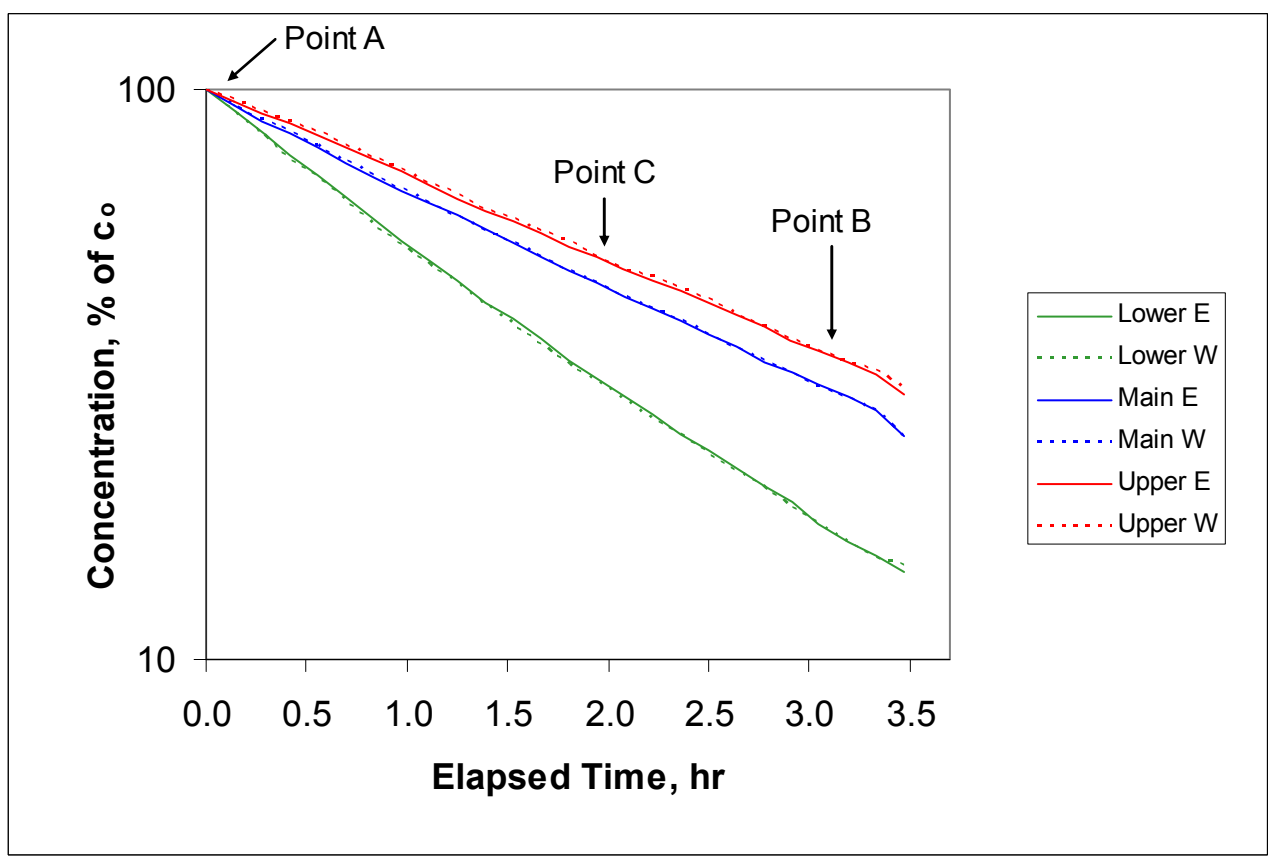

(b) Tracer decay curves in semilog format

Figure 11. Tracer gas decay curves for Field Example 1, showing very good mixing on each of the three levels of the home. 


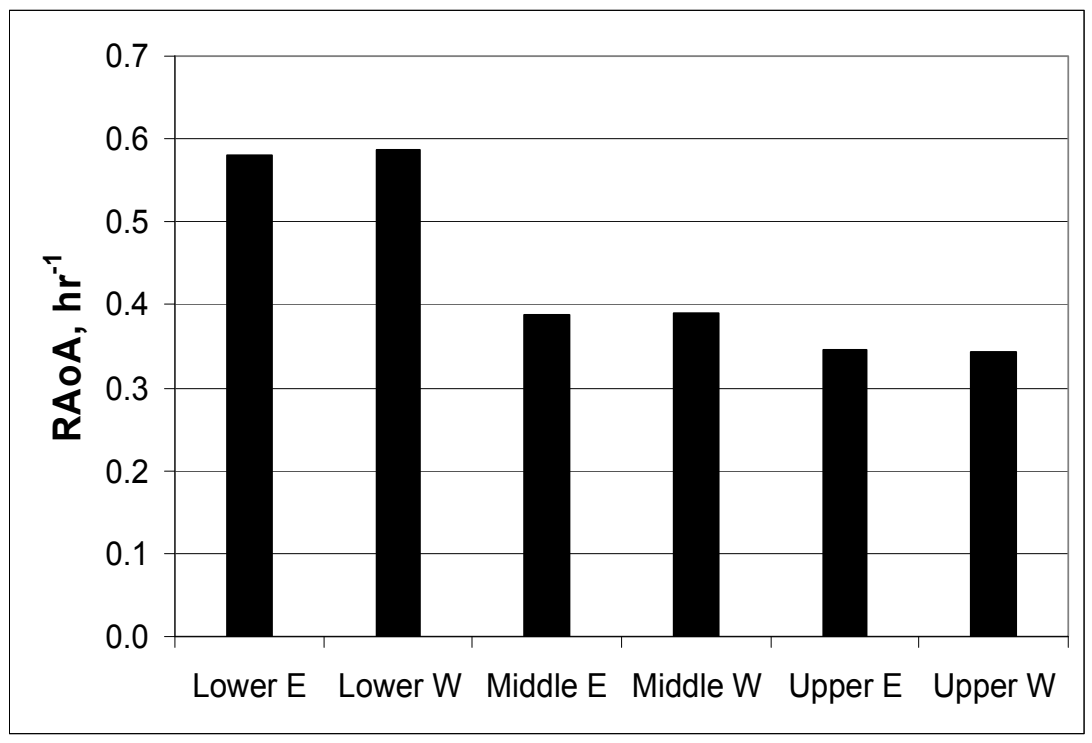

Figure 12. RAoA results for Field Example 1

\section{2 $\quad$ Field Example 2}

Figure 13 shows tracer decay data for a one-story, four-bedroom, 2,075- $\mathrm{ft}^{2}$ home in Sacramento, California, tested in December 2005 during relatively mild overnight winter weather conditions. The outdoor temperature ranged from $48^{\circ} \mathrm{F}$ to $50^{\circ} \mathrm{F}$, and the wind speed ranged from 0.4 to $4.4 \mathrm{mph}$ during the test. The indoor temperature was held constant at $72^{\circ} \mathrm{F}$. The mechanical ventilation system under test is a forced-air-integrated supply system, in which outside air is drawn into the central air return duct near the air handler via a smaller duct routed to an outside air inlet vent, adjusted to deliver $58 \mathrm{cfm}$. A timer controls the air handler blower when neither heating nor cooling is needed, ${ }^{9}$ on a duty cycle of 10 minutes on and 20 minutes off. The test was conducted with room doors closed. Further details of this field test are reported by Hendron et al. (2007), in which this is Case A6. We observe in Figure 13 that:

- The six decay curves, measured in different rooms, are very closely clustered, indicating a well-mixed condition in the home.

- The semilog graph is linear over the duration of the test, indicating a simple exponential function and steady conditions.

This situation resembles Analytical Example 1 (Section 5, Figure 4). Because the decay curve is a single, simple exponential function, we choose the Air-Change Rate method and apply Equation 28 (Section 7.3.1):

\footnotetext{
${ }^{9}$ As per the test protocol, portable electric space heaters were used to heat the home, so the air handler was controlled only by the timer during the test.
} 


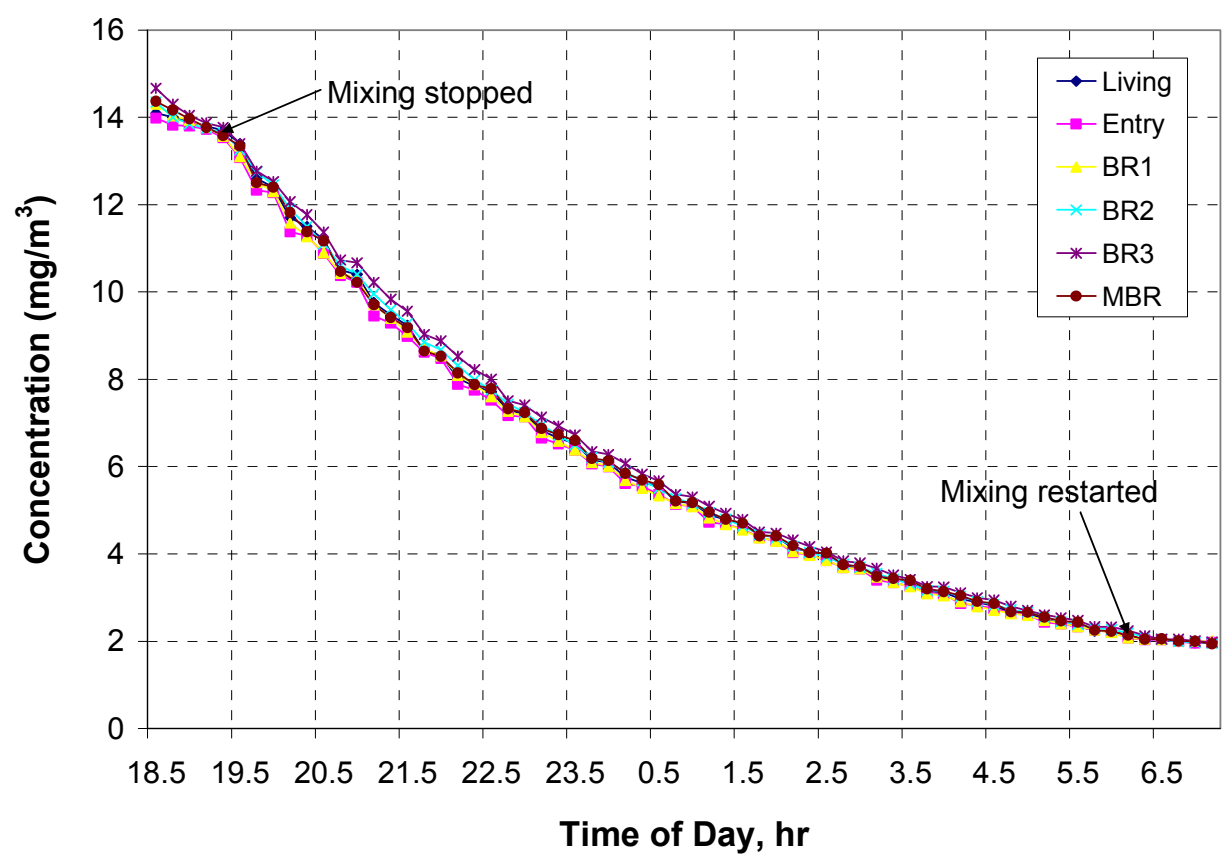

(a) Tracer decay curves in normal (arithmetic) format

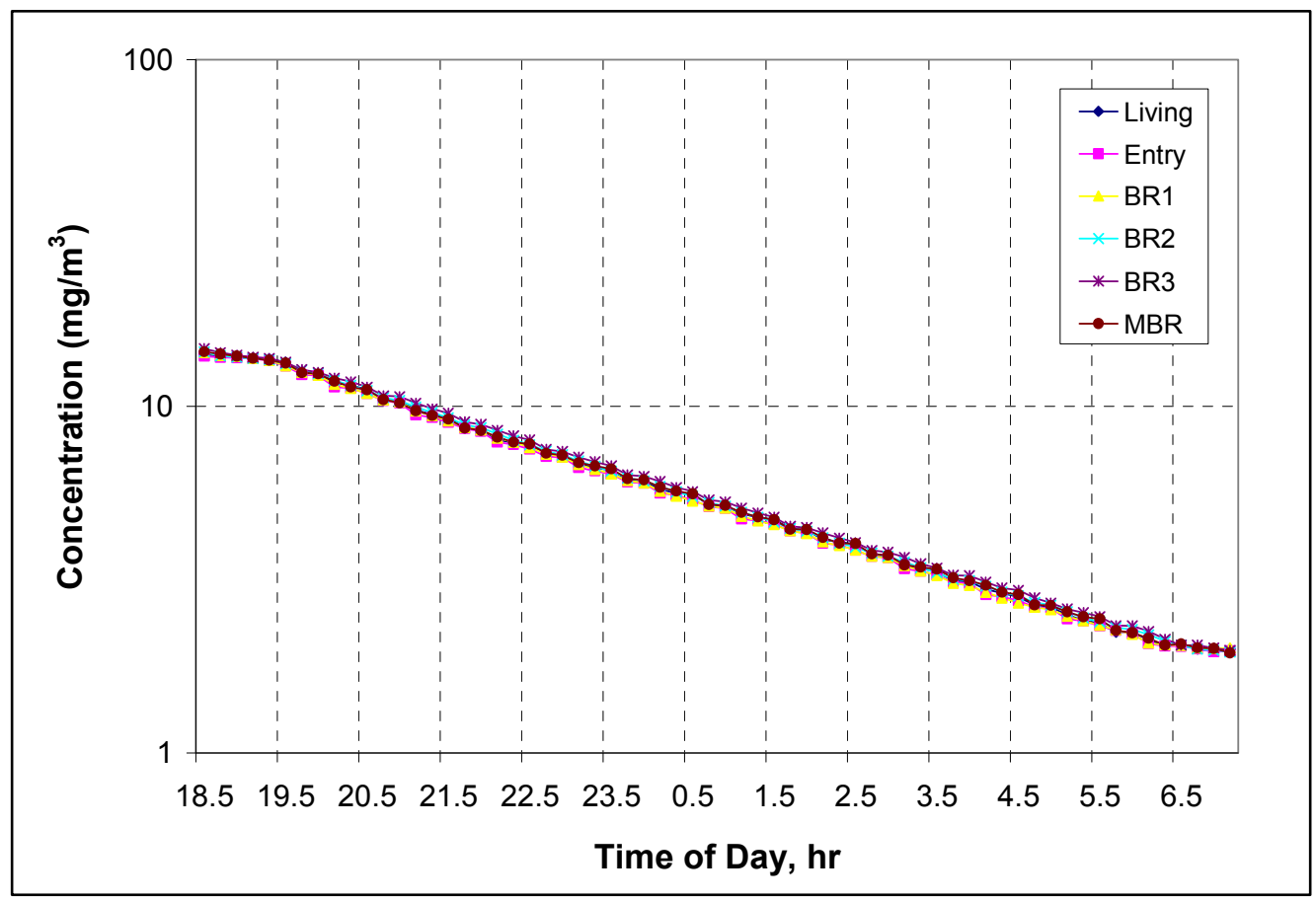

(b) Tracer decay curves in semilog format

Figure 13. Tracer gas decay curves for Field Example 2, showing steady, well-mixed conditions 


$$
A C R=\frac{\ln \left(c_{A} / c_{B}\right)}{T_{B}-T_{A}}
$$

Table 2. Data points for ACR calculation

\begin{tabular}{|c|c|c|}
\hline Point & Time (hr) & Concentration $^{\mathbf{1 0}}\left(\mathbf{m g} / \mathbf{m}^{\mathbf{3}}\right)$ \\
\hline A & 19.52 & 13.26 \\
\hline B & $24+6.11$ & 2.05 \\
\hline
\end{tabular}

Based on the data points shown in Table 2, we calculate the ACR as:

$$
A C R=\frac{\ln (13.26 / 2.05)}{(24+6.11)-19.52}=0.18 A C H
$$

This example demonstrates the uniform distribution of outside air provided by the forced-airintegrated ventilation system.

\subsection{Field Example 3}

Figure 14 shows tracer decay data for a one-story (plus finished basement), three- or four-bedroom, $2,950-\mathrm{ft}^{2}$ (including basement) home near Indianapolis, Indiana, that was tested in June 2001.

During the test, the outdoor temperature varied between $67^{\circ} \mathrm{F}$ and $71^{\circ} \mathrm{F}$, and the indoor temperature ranged from $67^{\circ} \mathrm{F}$ to $69^{\circ} \mathrm{F}\left(65^{\circ} \mathrm{F}\right.$ in the basement). The wind speed ranged from 0.0 to $2.5 \mathrm{mph}$. The ventilation system is a Broan Air Exchanger, which supplies tempered air to the main air return near the air handler. The Air Exchanger is designed to supply $60 \mathrm{cfm}$ of fresh air and temper it with $250 \mathrm{cfm}$ of recirculated air. Based on the total house volume and design airflow rate, the design ACR is $0.13 \mathrm{ACH}$. Room doors were closed during the test. We observe in Figure 14 that:

- There is significant separation among the decay curves, indicating unequal outside air distribution among the rooms.

- The great room and the kitchen receive significantly more tracer gas dilution than the basement, study, and bedrooms.

- Some of the curves are irregular, indicating non-steady conditions during the test.

Because of the non-steady test conditions, we apply the Graph Overlay Method instead of age-ofair analysis. The basic grid format shown in Figure 10 (Section 6) is adapted to this case by placing four reference curves to bracket the data. Based on inspection of the curves, we estimate the RAoA in the great room and kitchen as between $0.08 / \mathrm{hr}$ and $0.20 / \mathrm{hr}$. (In the semilog plot in Figure 14b, between hours 4 and 6, the slope of the decay curves appears to match the slope of the $0.08 / \mathrm{hr}$ grid curve, so we would not expect the decay curves to cross that grid curve if extended further in time.)

\footnotetext{
${ }^{10}$ Each value of concentration is an average across the 6 sample points.
} 


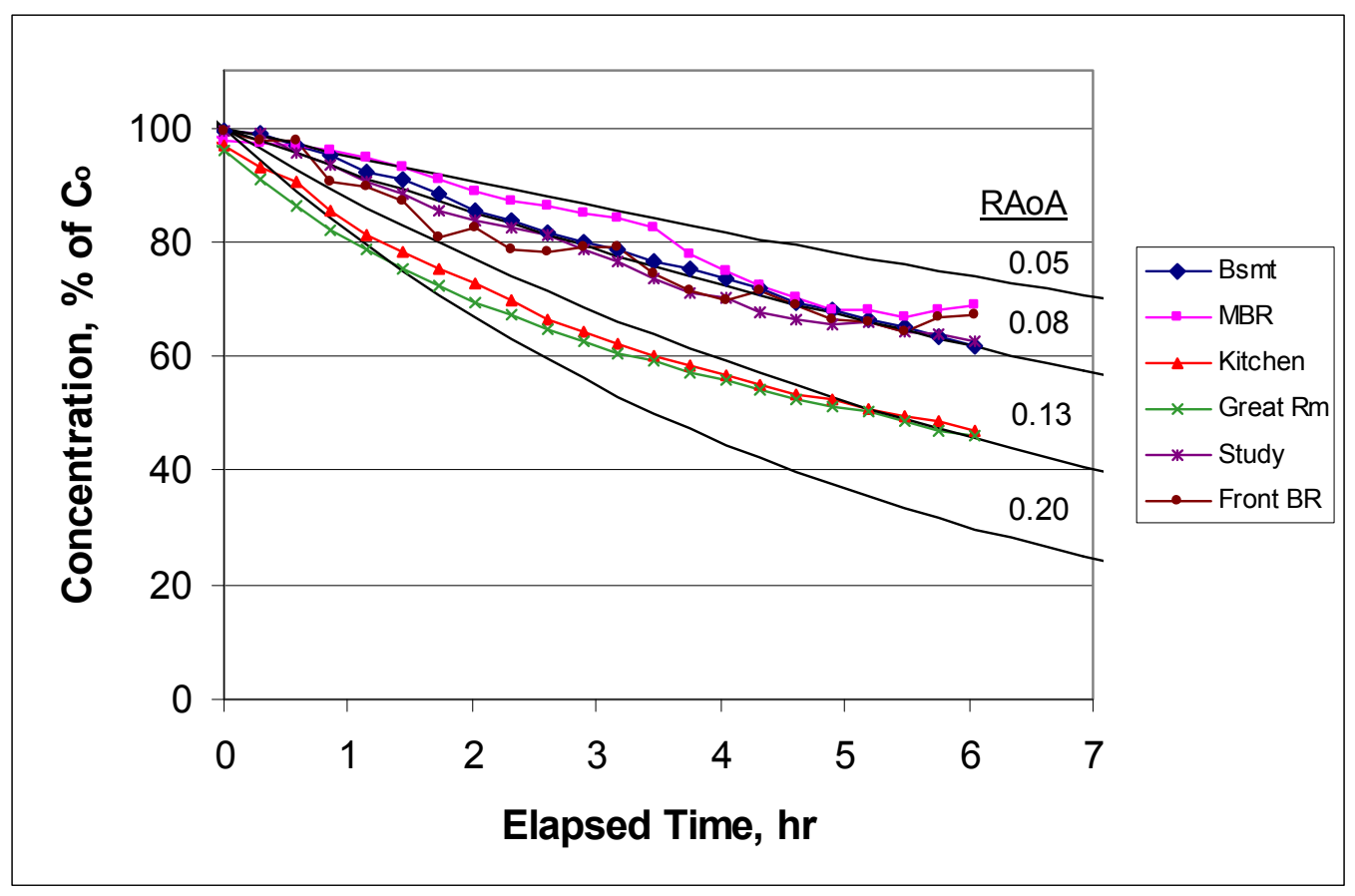

(a) Tracer decay curves in normal (arithmetic) format

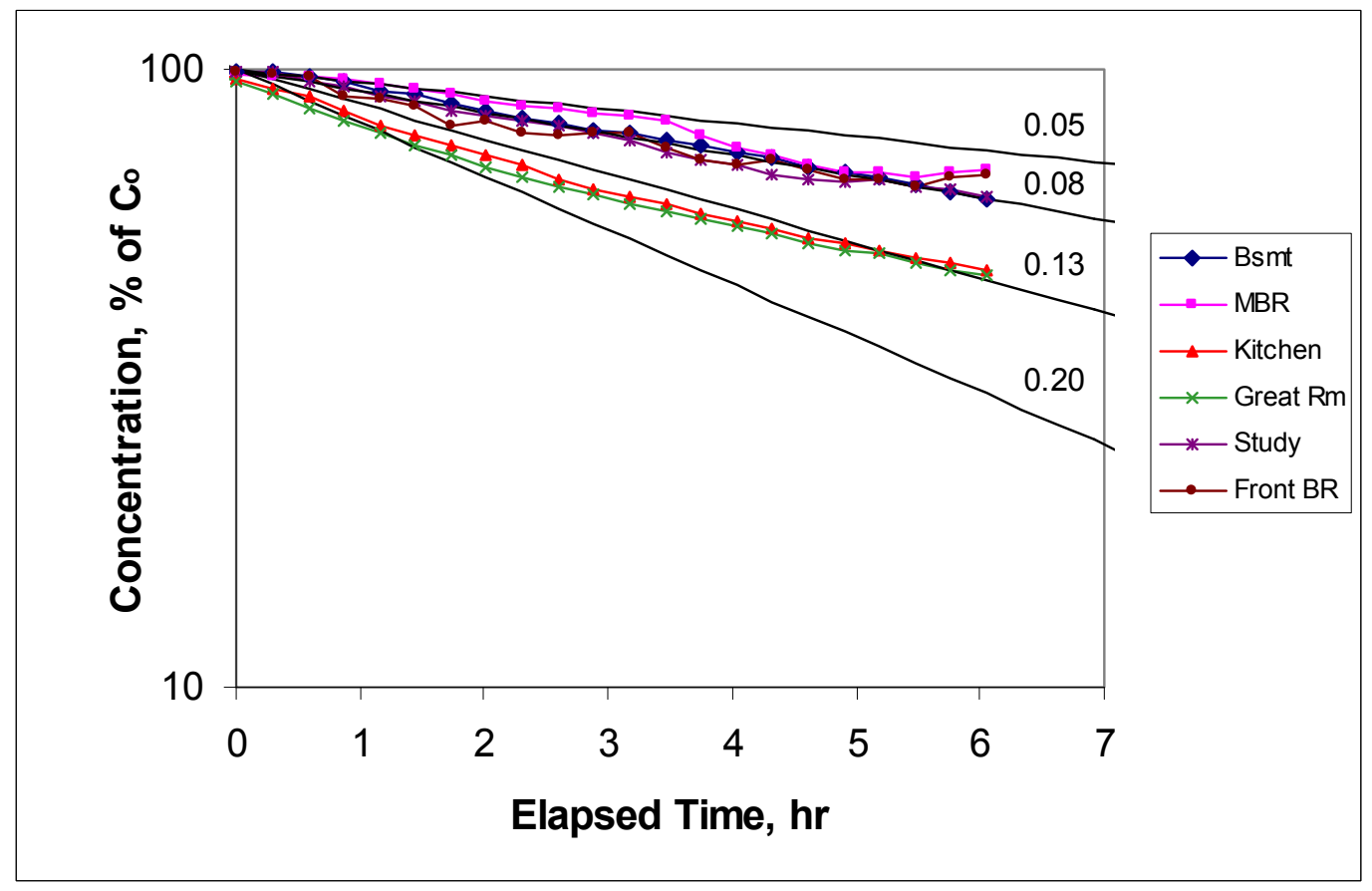

(b) Tracer decay curves in semilog format

Figure 14. Tracer gas decay curves for Field Example 3, showing unsteady, unmixed conditions 
We estimate the RAoA in the basement, study, and bedrooms as between $0.05 / \mathrm{hr}$ and $0.13 / \mathrm{hr}$. The conclusions drawn from this test are:

- The ventilation system does not distribute air uniformly throughout the home.

- Parts of the house (basement, study, and bedrooms) receive significantly less outside air than the design value of $0.13 / \mathrm{hr}$.

- The overall supply of outside air to the house is significantly less than the design value of $0.13 \mathrm{ACH}$. A likely cause of the shortfall is short circuiting of air through the duct system when the air handler is not running.

This example illustrates how our test protocol can provide meaningful results, even if conditions are not sufficiently steady to enable age-of-air analysis. See Section 8.7 for further discussion of test conditions related to steady and unsteady behavior.

\subsection{Field Example 4}

Figure 15 shows tracer decay data for a one-story, four-bedroom, 2,075- $\mathrm{ft}^{2}$ home in Sacramento, California (the same home featured in Field Example 2). This is a test of natural infiltration with no mechanical ventilation in operation. The test was conducted in January 2006, during the daytime, with room doors closed. The outdoor temperature was between $46^{\circ} \mathrm{F}$ and $51^{\circ} \mathrm{F}$, and the wind speed ranged from 0.4 to $5.1 \mathrm{mph}$ during the test. The indoor temperature was held constant at $72^{\circ} \mathrm{F}$. Further details of this field test are reported by Hendron et al. (2007), in which this is Case A15.

Although the curves do not appear erratic as in Example 3, they are irregular. The airflow conditions apparently changed at about hour 4. Most notably, one group of curves that was closely clustered from hour 0 to hour 4 begins to diverge after hour 4 . This anomaly disqualifies the test for age-of-air analysis. Instead, we apply the Graph Overlay Method, and estimate RAoA in all the rooms as between $0.05 / \mathrm{hr}$ and $0.13 / \mathrm{hr}$. Also, Bedroom 1 (BR1) receives somewhat less dilution effect than the other rooms. 


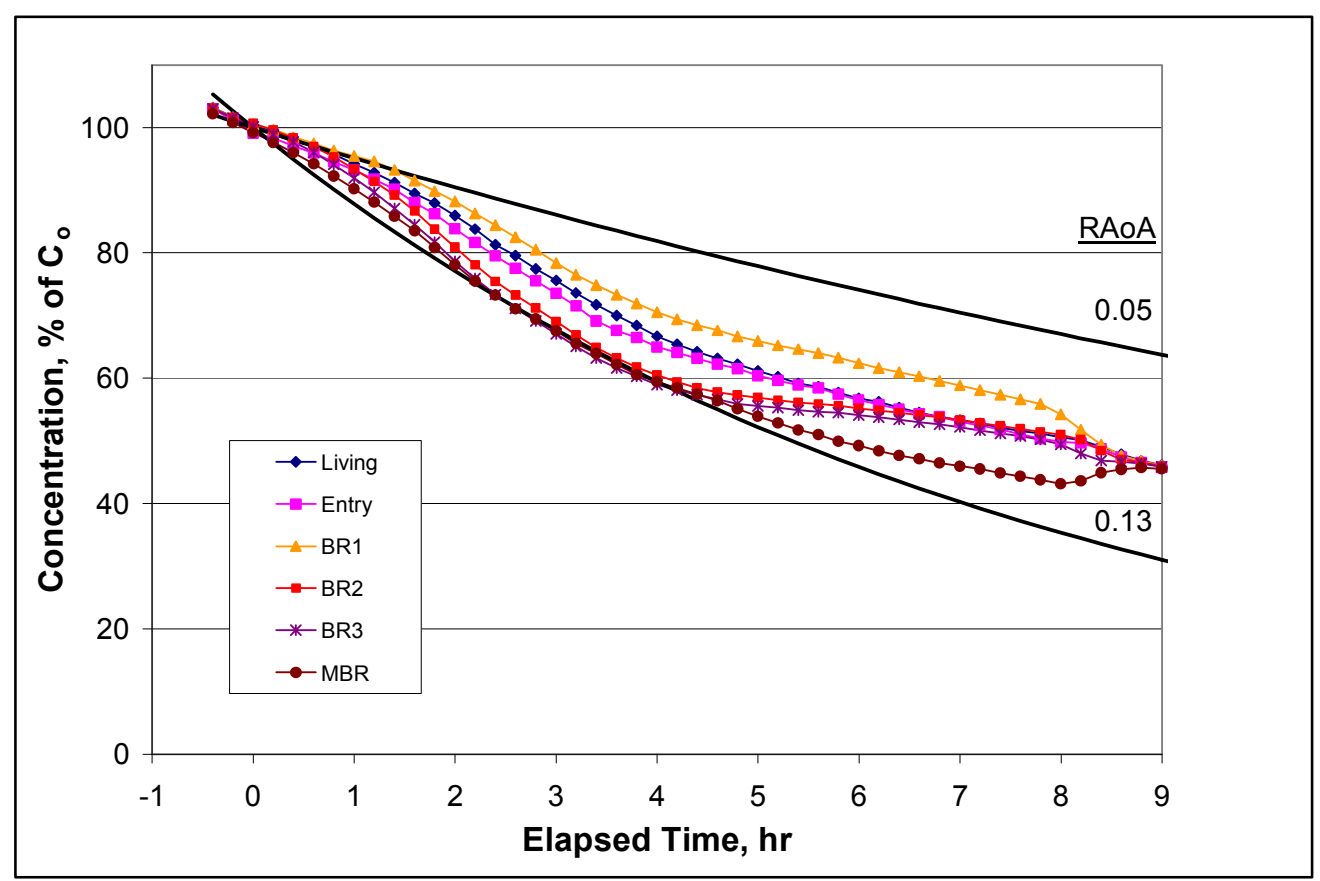

(a) Tracer decay curves in normal (arithmetic) format

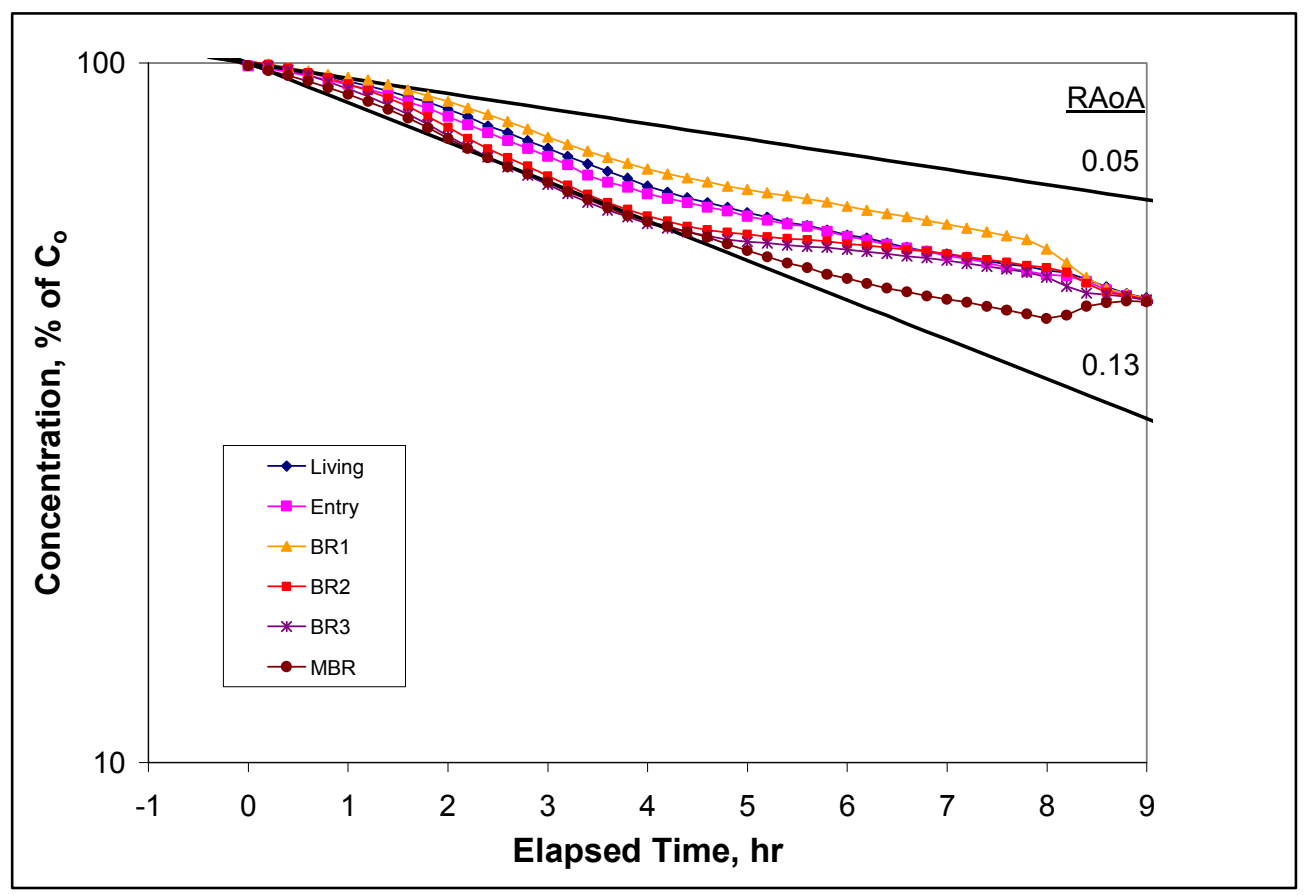

(b) Tracer decay curves in semilog format

Figure 15. Tracer gas decay curves for Field Example 4, showing unsteady, unmixed conditions 


\subsection{Field Example 5}

Figure 16 shows tracer decay data for a one-story, four-bedroom, 2,075- $\mathrm{ft}^{2}$ home in Sacramento, California (the same home featured in Field Examples 2 and 4). This test of a single-point exhaust ventilation system, adjusted to $58 \mathrm{cfm}$, was conducted overnight in December 2005, with room doors closed. The outdoor temperature ranged from $42^{\circ} \mathrm{F}$ to $56^{\circ} \mathrm{F}$ and the wind speed ranged from 0.4 to $2.7 \mathrm{mph}$ during the test. The indoor temperature was held constant at $72^{\circ} \mathrm{F}$. Further details of this field test are reported by Hendron et al. (2007), in which this is Case A3.

In this case, the curves appear sufficiently steady to enable age-of-air analysis. Conditions apparently changed slightly at about hour 0; in the semilog plot in Figure 16b, the slope of the Bedroom 2 (BR2) curve changes slightly. However, this anomaly is small compared to the differences among the curves, which are the object of the test. Point $C$ (described in Section 7.3.2) is shown at hour 2.4, after which the semilog curves appear linear through Point B. (Point C could have been designated as early as hour 1.0.) RAoA results based on these decay curves are shown in Figure 17. The highest RAoA occurs in the master bedroom. This result is logical, based on the two penetrations for exhaust fans in the master bath, which were turned off for this test in which the exhaust fan is in the laundry room was active. Thus, a large portion of the outside air entered the master bedroom directly. 


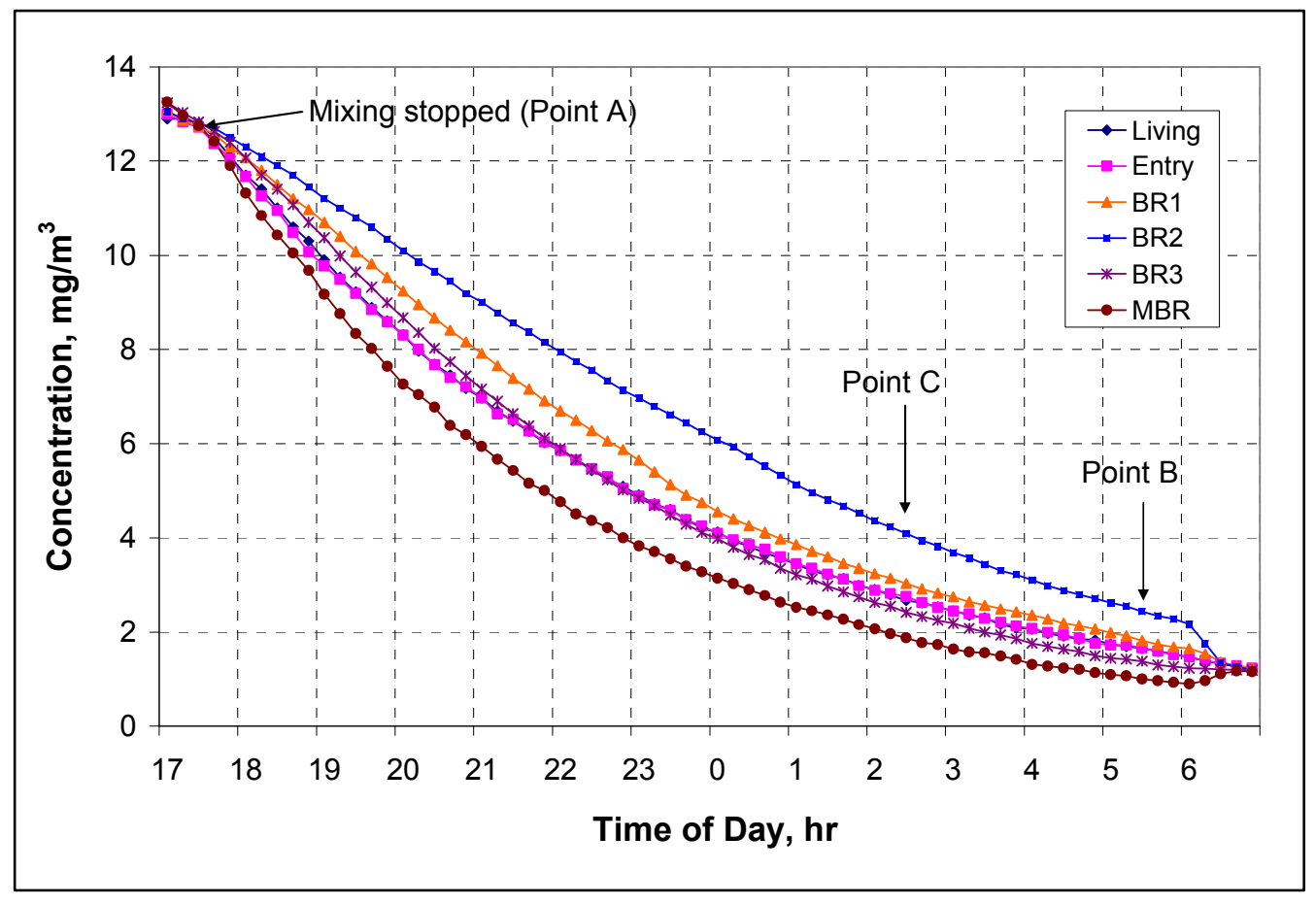

(a) Tracer decay curves in normal (arithmetic) format

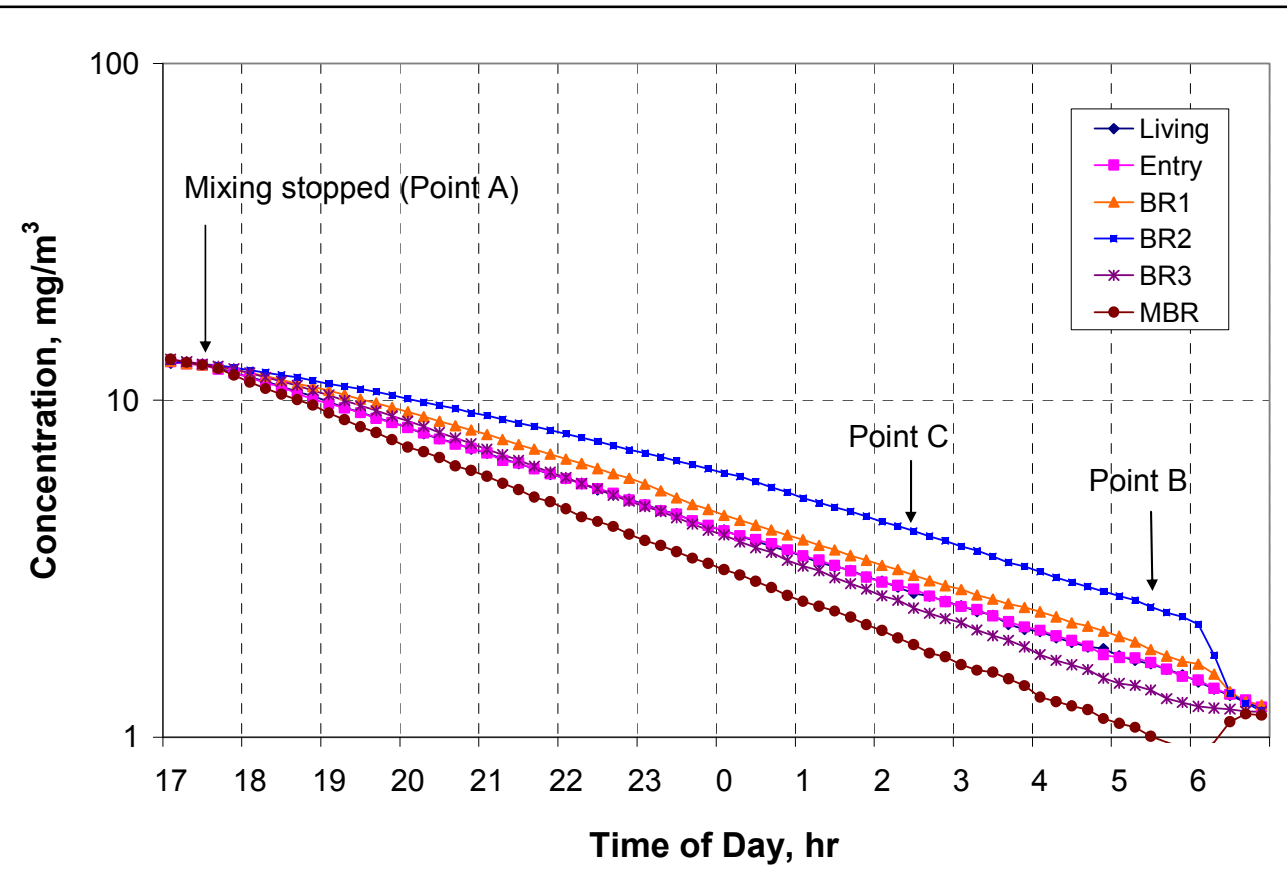

(b) Tracer decay curves in semilog format

Figure 16. Tracer gas decay curves for Field Example 5, showing steady, unmixed conditions 


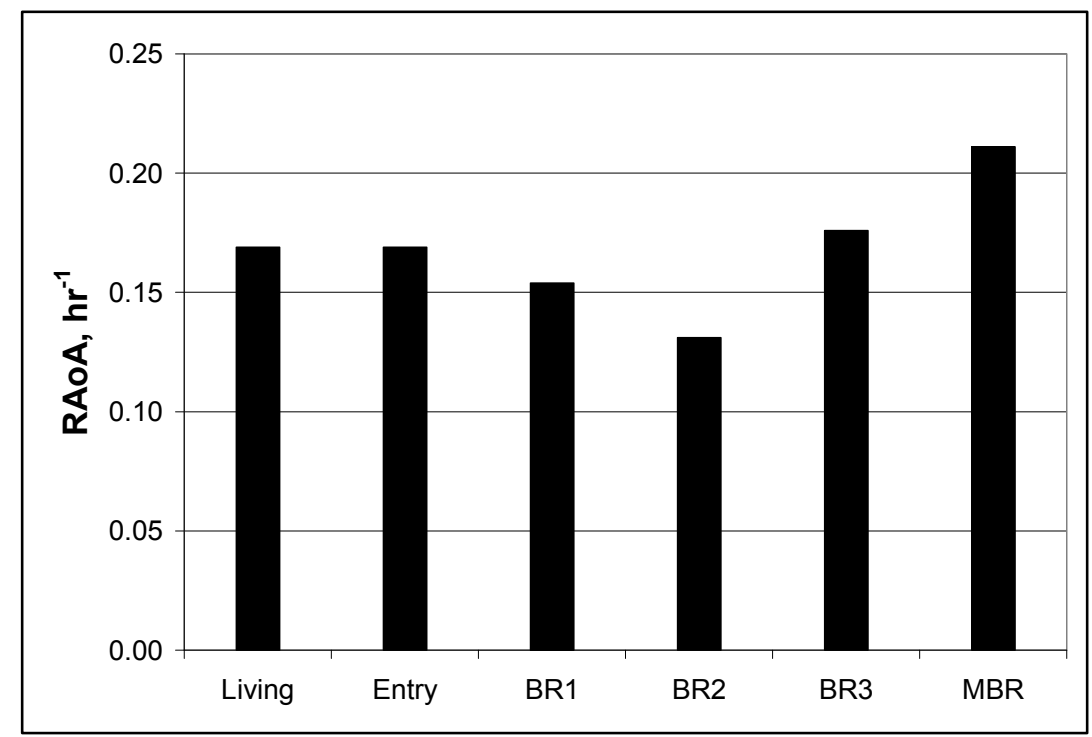

Figure 17. RAoA results for Field Example 5 


\subsection{Field Example 6}

Figure 18 shows tracer decay data for a two-story, four-bedroom, 2,582- $\mathrm{ft}^{2}$ home in Sacramento, California, tested in January 2006 during relatively mild overnight winter weather conditions. The outdoor temperature and wind speed were not recorded during this test. The indoor temperature was held constant at $72^{\circ} \mathrm{F}$. The mechanical ventilation system under test is a single-point exhaust fan located in the laundry room on the first floor, adjusted to $63 \mathrm{cfm}$. The test was conducted with room doors closed. Three sample points were selected on the first floor and three on the second floor. Further details of this field test are reported by Hendron et al. (2007), in which this is Case B1.

Comparing Figure 18a to Figures $6 \mathrm{~b}$ and $9 \mathrm{~b}$ (Section 5), the three upstairs rooms in this testBedroom 2 (BR2), Bedroom 3 (BR3), and the master bedroom (MBR) — exhibit behavior characteristic of partial series air flow. This is a typical result for the upper rooms in a multi-story home. Because of the stack effect, with the outdoor temperature lower than indoor temperature, outside air tends to enter the building near the bottom and leave the building near the top. Thus, the upstairs rooms receive airflow from the downstairs rooms. In the RAoA results shown below (Figure 19), the upstairs rooms show lower values of RAoA, related to air that has been in the house longer.

Based on the semilog curves in Figure 18b, test conditions may have changed at about hour 11 (the BR3 curve changes slope). Also, the BR1 curve becomes irregular at hour 12. Because the primary condition for ending the test is met at hour 11 (see Section 7.2.4), we designate this time as Point B. We designate hour 7 as Point $\mathrm{C}$ (Section 7.3.2), because the semilog curves appear linear between hour 7 and hour 11.

The initial mixing among the rooms at hour 0 is not as complete as in the previous examples. This affects the accuracy of the results, as should be noted when the results are presented. In this case, the difference is significantly smaller than the differences being measured among the various rooms, so we proceed with age-of-air analysis. RAoA results based on these decay curves are shown in Figure 19. The three rooms with the lowest values of RAoA are all on the second floor, as discussed above. 


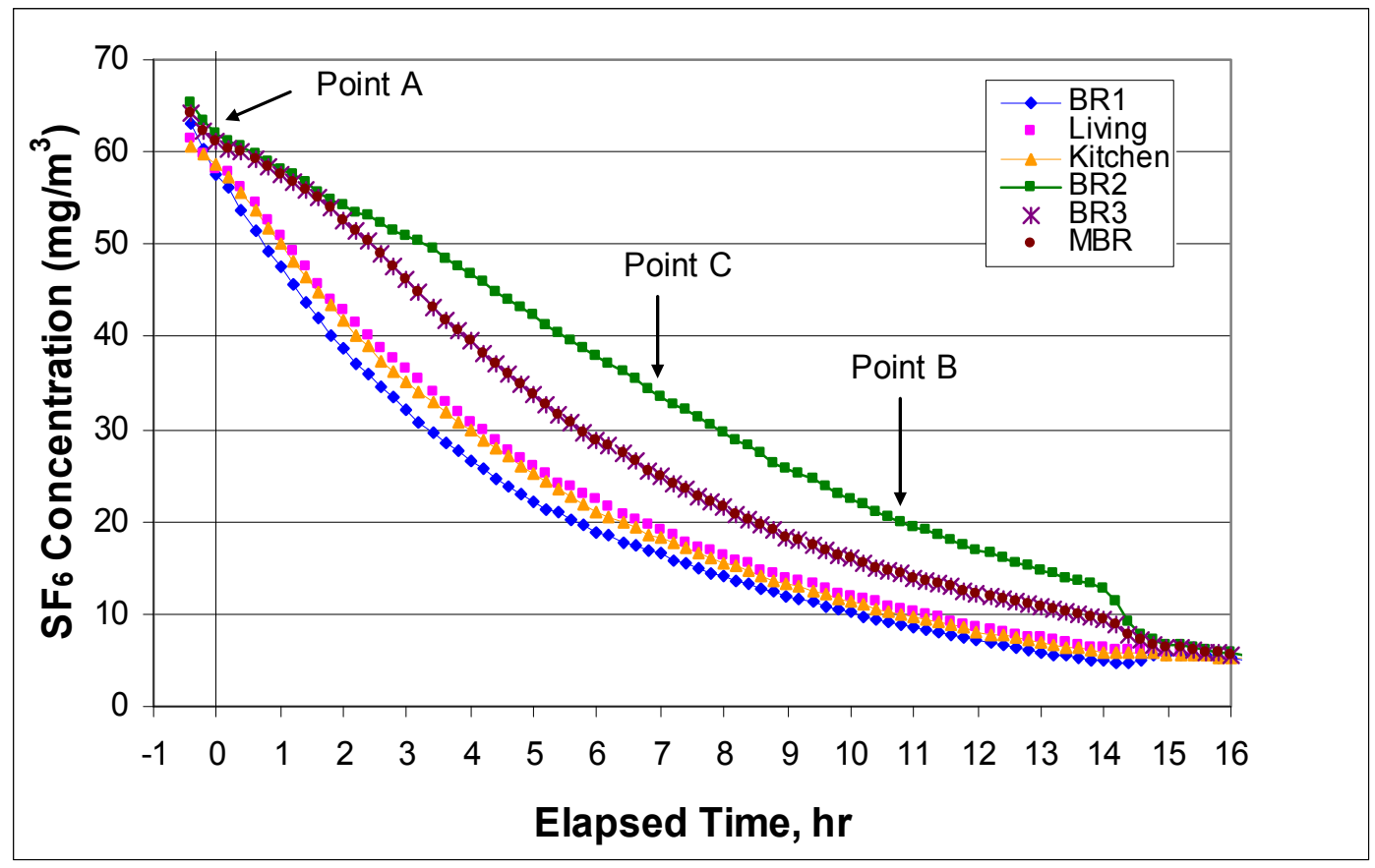

(a) Tracer decay curves in normal (arithmetic) format

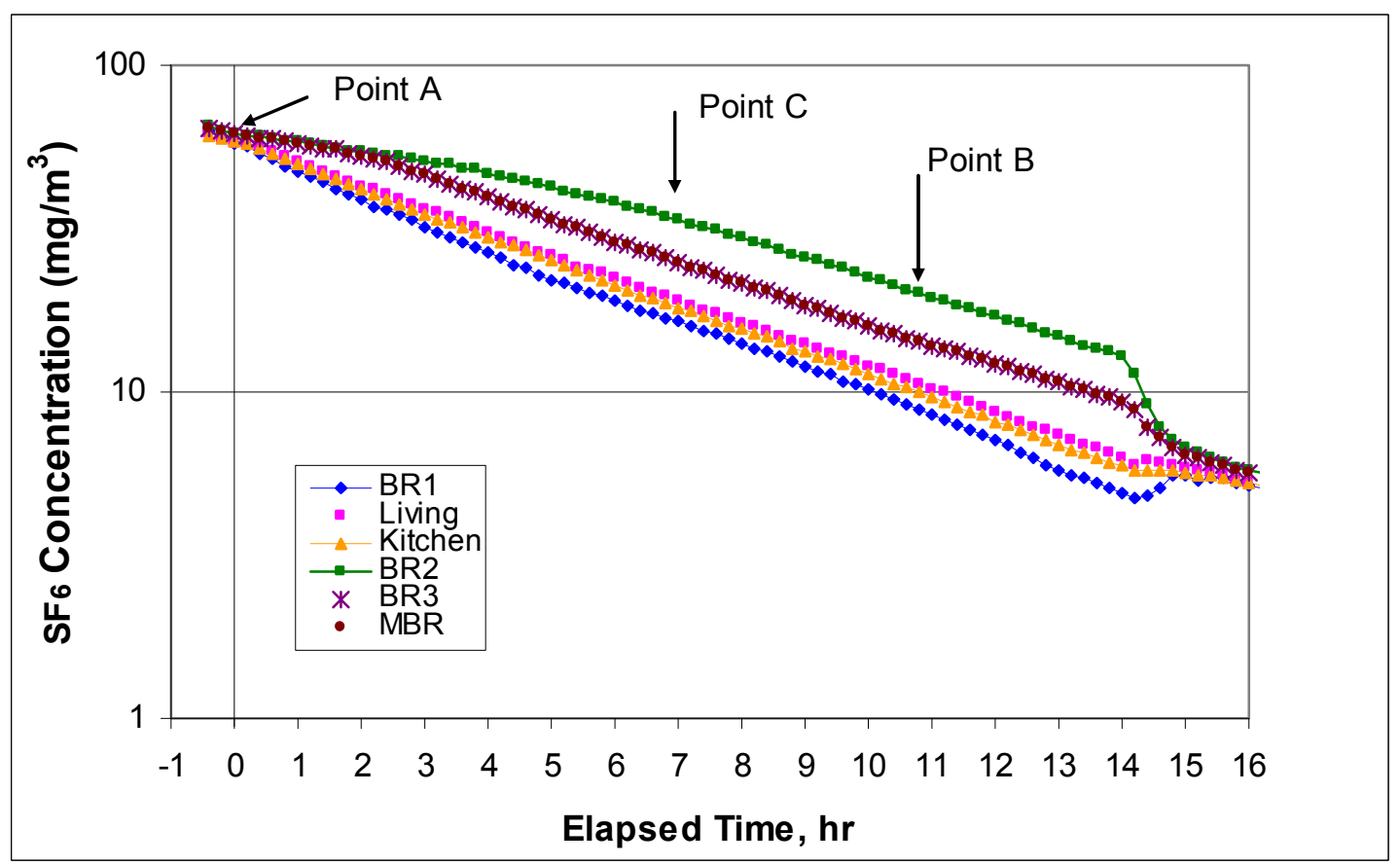

(b) Tracer decay curves in semilog format

Figure 18. Tracer gas decay curves for Field Example 6, showing steady, unmixed conditions 


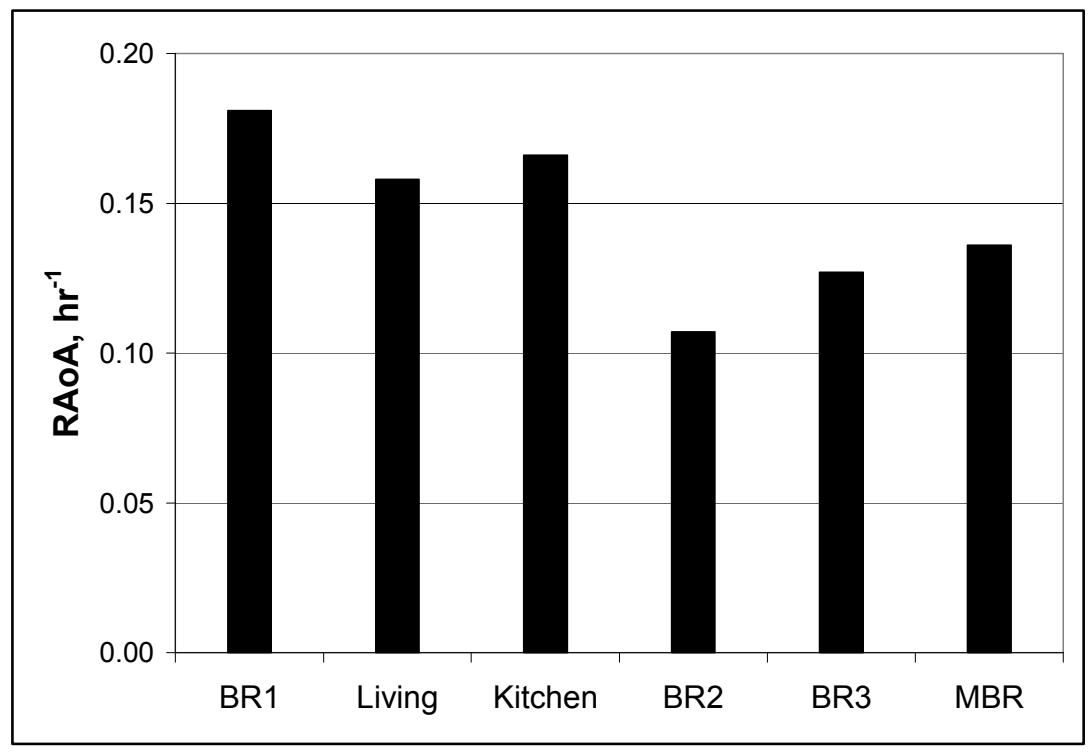

Figure 19. RAoA results for Field Example 6.

\subsection{Comparison of Test Conditions}

In the field tests described above, we found that conditions were sufficiently steady to conduct ageof-air analysis in Field Examples 1, 2, 5, and 6 (although we chose a different approach in Field Example 2). In Field Examples 3 and 4, we deemed the conditions unsteady for our purposes. In an effort to understand the test conditions under which sufficiently steady conditions can be expected, we compare the six cases in Table 3. The "Infiltration Indicators" shown in the table are rough estimates of the infiltration rate, based on the Sherman-Grimsrud model (Sherman and Modera 1986), using the peak measured wind speed and the average indoor-outdoor temperature difference. ${ }^{11}$ For the purpose of trying to predict steady or unsteady conditions, the amount of infiltration at the peak wind speed seems significant. We use the average indoor-outdoor temperature difference for this indicator, because in general the peak wind speed is not correlated with either the maximum or minimum temperature difference.

The house featured in case 1 is exceptionally tight, as indicated by the effective leakage area (ELA) of $29 \mathrm{in}^{2}$. All the other houses are roughly equal in leakage area. In case 4, the mechanical ventilation rate was $0 \mathrm{cfm}$ (this was a test of infiltration with no mechanical ventilation system in operation); the indoor-outdoor temperature difference was moderate; and the wind speed range was the highest of all the cases. It seems logical that the net air exchange rate was highly sensitive to the wind speed. In the steady cases with measured weather conditions (1, 2, and 5), the estimated infiltration rates are comparable to or smaller than the mechanical ventilation rates; thus, it seems logical that fluctuations in wind-driven infiltration were buffered somewhat by the mechanical ventilation. We do not understand the reason for the unsteady results in case 3. Compared to cases 2 and 5, case 3 has a somewhat smaller ELA, a similar mechanical ventilation rate, similar or milder weather conditions, and a lower estimated infiltration rate.

\footnotetext{
${ }^{11}$ We also assumed $25 \%$ of the leakage area in the floor, $25 \%$ of the leakage area in the ceiling, and wind shielding class IV.
} 
Table 3. Comparison of Test Conditions

\begin{tabular}{|c|c|c|c|c|c|c|c|c|}
\hline $\begin{array}{c}\text { Field } \\
\text { Example }\end{array}$ & Outcome & $\begin{array}{c}\text { No. of } \\
\text { Stories }\end{array}$ & $\begin{array}{c}\text { ELA } \\
\text { (in }^{\mathbf{2}} \text { ) }\end{array}$ & $\begin{array}{c}\text { Wind } \\
\text { Speed } \\
\text { (mph) }\end{array}$ & $\begin{array}{c}\text { Outdoor } \\
\text { Temp. } \\
\left({ }^{\circ} \mathbf{F}\right)\end{array}$ & $\begin{array}{c}\text { Indoor } \\
\text { Temp } \\
\text { ('F) }\end{array}$ & $\begin{array}{c}\text { Infiltration } \\
\text { Indicator } \\
\text { (cfm) }\end{array}$ & $\begin{array}{c}\text { Mechanical } \\
\text { Ventilation } \\
\text { Rate (cfm) }\end{array}$ \\
\hline 1 & Steady & 3 & 29 & 0.7 to 2.0 & 52 to 58 & 72 to 79 & 28 & 80 \\
\hline 2 & Steady & 1 & 92 & 0.4 to 4.3 & 48 to 50 & 72 & 64 & 58 \\
\hline 3 & Unsteady & 1 & 80 & 0.0 to 2.5 & 67 to 71 & 67 to 69 & 20 & 60 \\
\hline 4 & Unsteady & 1 & 92 & 0.4 to 5.1 & 46 to 51 & 72 & 68 & 0 \\
\hline 5 & Steady & 1 & 92 & 0.4 to 2.7 & 42 to 56 & 72 & 58 & 58 \\
\hline 6 & Steady & 2 & 80 & Unknown & Unknown & 72 & Unknown & 63 \\
\hline
\end{tabular}

Possible explanations include:

- Shielding of the anemometer by surrounding buildings or trees. ${ }^{13}$

- An unusual sensitivity to wind speed caused by local terrain or the orientation of openings (e.g., building envelope cracks or the mechanical ventilation intake) relative to the wind direction.

- An error in recording the test conditions, which occurred more than 6 years ago.

Based on the limited experience of these examples, we can expect steady conditions when the infiltration rate is smaller than or comparable to the mechanical ventilation rate. Tests of natural infiltration alone are less likely to be amenable to age-of-air analysis.

\footnotetext{
${ }^{12}$ For accurate measurement of wind speed, an anemometer should be placed on a tower well above surrounding objects. This is generally not practical in our field testing situations. Typically, the anemometer is placed where shielding by surrounding buildings (including the building under test) is significant, so the measured wind speeds provide only a rough indication of the weather conditions.

${ }^{13}$ See previous footnote.
} 


\section{Summary and Conclusions}

A test and analysis protocol has been developed as a practical approach for measuring outside air distribution in homes. We recommend the use of this protocol within the context of the Building America field testing program. We address the issue of unknown occupant behavior through the use of RAoA as a surrogate for air quality, based on how long the air has been within the building. We address the issue of steady-state conditions, required for age-of-air analysis, by (1) planning field tests for mild, steady weather to the extent possible, (2) reviewing the data with engineering judgment, and (3) providing an alternative, semi-quantitative method of analysis (the Graph Overlay Method) for use when the airflows are not sufficiently steady. This test and analysis protocol has been used successfully in a number of field tests and has led to significant insights on ventilation design issues. Performance advantages of more sophisticated ventilation systems over simpler, less costly designs have been verified, and specific problems, such as airflow shortcircuiting, have been identified. The lessons learned from this test approach can make a significant contribution to the attainment of the energy conservation and occupant health and comfort goals of the Building America program.

The results of the field examples presented in Section 8 should not be interpreted as representing the current state of the art in residential ventilation systems. These field tests were experimental in nature, and the examples were selected to illustrate particular analysis issues. A more comprehensive set of field test results is needed to evaluate current builder practice with respect to outside air distribution in homes.

\section{Acknowledgments}

The authors appreciate the participation of Armin Rudd and Aaron Townsend of Building Science Corporation (BSC) in field test exercises and valuable discussions that have helped to shape and clarify the test and analysis protocol. Bill Rittelmann of Integrated Building and Construction Solutions (IBACOS) was very helpful in planning and conducting early field tests with this approach. Craig Christensen of NREL, in addition to volunteering the use of his home, also provided significant input to the test methodology. Robb Aldrich of Steven Winter Associates (SWA) and Brent Griffith of NREL provided peer reviews that have improved this report. This work was performed with the support of Ed Pollock, George James, and Terry Logee of the DOE Residential Buildings program. 


\section{References}

ASHRAE (2002). ANSI/ASHRAE Standard 129-1997 (RA 2002). Measuring Air-Change Effectiveness. Atlanta, GA: American Society of Heating, Refrigerating and Air-Conditioning Engineers, Inc.

ASHRAE (2004). Ventilation for Acceptable Indoor Air Quality, ANSI/ASHRAE Standard 62.12004. Atlanta, GA: American Society of Heating, Refrigerating and Air-Conditioning Engineers, Inc.

ASHRAE (2005). Handbook-Fundamentals, p. 27.1. Atlanta, GA: American Society of Heating, Refrigerating and Air-Conditioning Engineers, Inc.

ASHRAE (2007). Ventilation and Acceptable Indoor Air Quality in Low-Rise Residential Buildings, ANSI/ASHRAE Standard 62.2-2007. Atlanta, GA: American Society of Heating, Refrigerating and Air-Conditioning Engineers, Inc.

ASTM (2004). Standard Guide for Specifying and Evaluating Performance of Single Family Attached and Detached Dwellings-Indoor Air Quality, E2267-04. West Conshohocken, PA: ASTM International.

Brüel and Kjær, Nærum, Denmark. http://www.bksv.com/, last accessed August 29, 2007.

CSA (2003). CAN/CSA F326-M91 Residential Mechanical Ventilation Systems. Canadian Standards Association, Ontario, Canada. http://www.csaintl.org/onlinestore/GetCatalogItemDetails.asp?mat=2410793\&Parent=102, last accessed August 29, 2007.

Dietz, R.N.; Goodrich, R.W.; Cote, E.A.; Wieser, R.F. (1986). "Detailed Description and Performance of a Passive Perfluorocarbon Tracer System for Building Ventilation and Air Exchange Measurements." Measured Air Leakage of Buildings, ASTM STP 904, H.R. Trechsel and P.L. Lagus, eds. Philadelphia, PA: American Society for Testing and Materials, pp. 203264.

Fortmann, R.C.; Nagda, N.L.; Rector, H.E. (1990). "Comparison of Methods for the Measurement of Air-Change Rates and Interzonal Airflows to Two Test Residences." In Air Change Rate and Airtightness in Buildings, ASTM STP 1067. M.H. Sherman, ed. West Conshohocken, PA: American Society for Testing and Materials, pp. 104-118.

Grieve, P.W. (1991). Measuring Ventilation Using Tracer-Gases. Denmark: Brüel \& Kjær.

Harrje, D.T.; Dutt, G.S.; Bohac, D.L.; Gadsby, K.J. (1985). Documenting air movements and infiltration in multicell buildings using various tracer techniques. ASHRAE Transactions 91(2): 2012-2027.

Harrje, D.T.; Dietz, R.N.; Sherman, M.; Bohac, D.L.; D’Ottavio, T.W.; Dickerhoff, J.J. (1990). "Tracer Gas Measurement Systems Compared in a Multifamily Building." In Air change rate and airtightness in buildings, ASTM STP 1067. M.H. Sherman, ed. West Conshohocken, PA: American Society for Testing and Materials, pp. 5-12.

Hendron, R. (2006). Building America Benchmark Definition, Updated December 15, 2006. NREL TP-550-40968. Golden, CO: National Renewable Energy Laboratory.

http://www.nrel.gov/docs/fy07osti/40968.pdf, last accessed November 26, 2007. 
Hendron, R.; Rudd, A.; Anderson, R.; Barley, D.; Hancock, E.; Townsend, A. (2007). "Field Test of Room-to-Room Distribution of Outside Air with Two Residential Ventilation Systems," TOPC-00106-2007.R1, ASHRAE IAQ 2007 Conference, Baltimore, MD, Oct. 14-17. Atlanta, GA: American Society of Heating, Refrigerating and Air-Conditioning Engineers, Inc.

IPCC (2001). Climate Change 2001: A Scientific Basis, Intergovernmental Panel on Climate Change; Houghton, J.T.; Ding, Y.; Griggs, D.J.; Noguer, M.; van der Linden, P.J.; Dai, X.; Johnson, C.A.; and Maskell, K. eds., Cambridge, U.K.: Cambridge University Press.

McWilliams, J.; Sherman, M. (2005). Review of Literature Related to Residential Ventilation Requirements, Report No. LBNL-57236. Berkeley, CA: Lawrence Berkeley National Laboratory.

MN Chapter 7672. Minnesota Energy Code Chapter 7672, Detached Single-Family And TwoFamily Dwellings. http://www.state.mn.us/mn/externalDocs/Commerce/Chapter_7672,_Residential_11130201242 8 72rule.pdf, last accessed August 29, 2007.

Palmiter, L.; Bond, T. (1991). Modeled and Measured Infiltration. EPRI CU-7327. Palo Alto, CA: Electric Power Research Institute.

Persily, A.K.; Martin, S.R. (2000). A Modeling Study of Ventilation in Manufactured Houses. Gaithersburg, MD: National Institute of Standards and Technology; NISTIR 6455.

Reardon, J.T.; Shaw, C. (1997). "Evaluation of Five Simple Ventilation Strategies Suitable for Houses without Forced-Air Heating.” ASHRAE Transactions Vol. 103, Pt 1, pp. 731-744.

Rudd, A.F., and Lstiburek, J.W. (2000). "Measurement of Ventilation and Interzonal Distribution in Single-Family Homes." ASHRAE Transactions 2000, MN-00-10-3, Vol. 106, Pt. 2. Atlanta, GA: American Society of Heating, Refrigeration and Air-Conditioning Engineers.

Sandberg, M. (1981). "What Is Ventilation Efficiency?" Building and Environment, Vol. 16, No. 2, pp. 123-135.

Sherman, M.H.; Dickerhoff, D. (1989). “A Multi-Gas Tracer System for Multi-Zone Airflow Measurements." Proceedings of the ASHRAE/DOE/BTECC/CIBSE Conference-Thermal Performance of the Exterior Envelopes of Buildings IV; pp. 417-432.

Sherman, M.H.; Modera, M.P. (1986). "Comparison of Measured and Predicted Infiltration Using the LBL Infiltration Model," Measured Air Leakage of Buildings, ASTM STP 904, H. R. Trechsel and P. L. Lagus, Eds., pp. 325-347. Philadelphia, PA: American Society for Testing and Materials.

Sinden, F.W. (1978). "Multi-Chamber Theory of Air Infiltration.” Building and Environment 13:21-28.

WAC (2004). Washington State Title 51 WAC Community, trade, and economic development, department of (building code council), Chapter 51-13 Ventilation and indoor air quality. http://apps.leg.wa.gov/WAC/default.aspx?cite=51-13, last accessed September 21, 2007.

Wortman, D.; Burch, J.; Judkoff, R. (1982). “A Multizone Infiltration Monitoring System.” In Progress in Passive Solar Energy Systems, Proceedings of the 7th National Passive Conference, Knoxville, TN, August 29 to September 1, pp. 767-771. Boulder, CO: American Solar Energy Society. Also SERI/TP-254-1638. Golden, CO: National Renewable Energy Laboratory. 


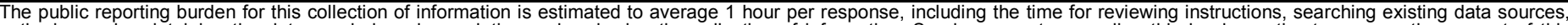

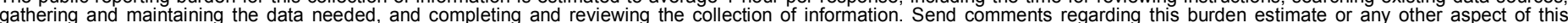

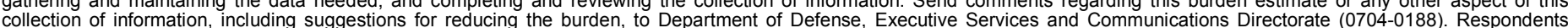

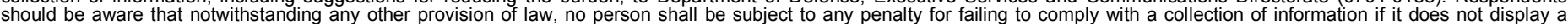

should be aware that notwithstanding

PLEASE DO NOT RETURN YOUR FORM TO THE ABOVE ORGANIZATION.

\begin{tabular}{l|l|l|l} 
1. REPORT DATE $(D D-M M-Y Y Y Y)$ & 2. & REPORT TYPE & 3. DATES COVERED (FrOm - TO)
\end{tabular}

December 2007

Technical Report

4. TITLE AND SUBTITLE

Test Protocol for Room-to-Room Distribution of Outside Air by

Residential Ventilation Systems 5a. CONTRACT NUMBER

DE-AC36-99-G010337

5b. GRANT NUMBER

5c. PROGRAM ELEMENT NUMBER

5d. PROJECT NUMBER

NREL/TP-550-31548

5e. TASK NUMBER

BET78004

5f. WORK UNIT NUMBER
7. PERFORMING ORGANIZATION NAME(S) AND ADDRESS(ES)

National Renewable Energy Laboratory

1617 Cole Blvd.

Golden, CO 80401-3393
8. PERFORMING ORGANIZATION REPORT NUMBER

NREL/TP-550-31548

9. SPONSORING/MONITORING AGENCY NAME(S) AND ADDRESS(ES)

10. SPONSOR/MONITOR'S ACRONYM(S) NREL

11. SPONSORING/MONITORING AGENCY REPORT NUMBER

12. DISTRIBUTION AVAILABILITY STATEMENT

National Technical Information Service

U.S. Department of Commerce

5285 Port Royal Road

Springfield, VA 22161

13. SUPPLEMENTARY NOTES

14. ABSTRACT (Maximum 200 Words)

This test and analysis protocol has been developed as a practical approach for measuring outside air distribution in homes. It has been used successfully in field tests and has led to significant insights on ventilation design issues. Performance advantages of more sophisticated ventilation systems over simpler, less-costly designs have been verified, and specific problems, such as airflow short-circuiting, have been identified.

15. SUBJECT TERMS

outside air; airflow; air distribution; indoor air quality; building america

\begin{tabular}{l}
\hline \multicolumn{3}{|l|}{ 16. SECURITY CLASSIFICATION OF: } \\
\hline \begin{tabular}{l|l|l|} 
a. REPORT & b. ABSTRACT & c. THIS PAGE \\
Unclassified & Unclassified & Unclassified \\
& & \\
\hline
\end{tabular} \\
\hline
\end{tabular}

\begin{tabular}{l|l} 
17. LIMITATION \\
OF ABSTRACT \\
UL
\end{tabular}

19a. NAME OF RESPONSIBLE PERSON

19b. TELEPHONE NUMBER (Include area code) 


\section{A Strong Energy Portfolio for a Strong America}

Energy efficiency and clean, renewable energy will mean a stronger economy, a cleaner environment, and greater energy independence for America. Working with a wide array of state, community, industry, and university partners, the U.S. Department of Energy's Office of Energy Efficiency and Renewable Energy invests in a diverse portfolio of energy technologies.

\section{Research and Development of Buildings}

Our nation's buildings consume more energy than any other sector of the U.S. economy, including transportation and industry. Fortunately, the opportunities to reduce building energy useand the associated environmental impacts—are significant.

DOE's Building Technologies Program works to improve the energy efficiency of our nation's buildings through innovative new technologies and better building practices. The program focuses on two key areas:

\section{- Emerging Technologies} Research and development of the next generation of energy-efficient components, materials, and equipment

- Technology Integration Integration of new technologies with innovative building methods to optimize building performance and savings

For more information contact: EERE Information Center 1-877-EERE-INF (1-877-337-3463) www.eere.energy.gov

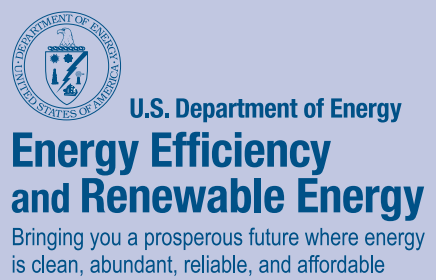

An electronic copy of this publication is available on the Building America Web site at www.buildingamerica.gov

\section{Visit our Web sites at:}

\section{Building
U.S. Department of Energy
Research Toward Zero Energy Homes}

Building America Program

George S. James • New Construction • 202-586-9472• fax: 202-586-8134• e-mail: George.James@ee.doe.gov

Terry Logee • Existing Homes • 202-586-1689 • fax: 202-586-4617• e-mail: terry.logee@ee.doe.gov

Lew Pratsch • Integrated Onsite Power • 202-586-1512 • fax: 202-586-8185 • e-mail: Lew.Pratsch@hq.doe.gov

Building America Program • Office of Building Technologies, EE-2J • U.S. Department of Energy • 1000 Independence Avenue, S.W. • Washington, D.C. 20585-0121・ www.buildingamerica.gov

Building Industry Research Alliance (BIRA)

Robert Hammon • ConSol • 7407 Tam 0'Shanter Drive \#200 • Stockton, CA 95210-3370 • 209-473-5000 • fax: 209-474-0817• e-mail: Rob@consol.ws•www.bira.ws

Building Science Consortium (BSC)

Betsy Pettit • Building Science Consortium (BSC) • 70 Main Street • Westford, MA $01886 \bullet 978-589-5100 \bullet$ fax: 978-589-5103 • e-mail: Betsy@buildingscience.com• www.buildingscience.com

Consortium for Advanced Residential Buildings (CARB)

Steven Winter • Steven Winter Associates, Inc. • 50 Washington Street • Norwalk, CT $06854 \bullet 203-857-0200 \bullet$ fax: 203-852-0741 • e-mail: swinter@swinter.com • www.carb-swa.com

\section{Davis Energy Group}

David Springer • Davis Energy Group • 123 C Street • Davis, CA 95616 • 530-753-1100 • fax: 530-753-4125• e-mail: springer@davisenergy.com • deg@davisenergy.com • www.davisenergy.com/index.html

IBACOS Consortium

Brad Oberg • IBACOS Consortium • 2214 Liberty Avenue • Pittsburgh, PA $15222 \bullet 412-765-3664 \bullet$ fax: 412-765-3738 • e-mail: boberg@ibacos.com • www.ibacos.com • IBACOS provided technical support for TNAH '07

Industrialized Housing Partnership (IHP)

Subrato Chandra • Florida Solar Energy Center • 1679 Clearlake Road • Cocoa, FL 32922 • 321-638-1412 • fax: 321-638-1439 • e-mail: subrato@fsec.ucf.edu • www.baihp.org • IHP provided on-site support for TNAH '07

National Council of the Housing Industry (NCHI) of the NAHB

Tucker Bernard • NCHI • The Supplier $100 \bullet$ National Association of Home Builders (NAHB) • 1201 15th St. NW, Washington, D.C. 20005 • 800-368-5242 ext. 8519• e-mail: tbernard@nahb.org • www.nahb.org

National Renewable Energy Laboratory

Ren Anderson • 1617 Cole Boulevard, MS-1725•Golden, C0 80401 • 303-384-7433• fax: 303-384-7540•

e-mail: ren_anderson@nrel.gov • www.nrel.gov

Tim Merrigan • 1617 Cole Boulevard, MS-1725 • Golden, C0 80401 • 303-384-7349 • fax: 303-384-7540 •

e-mail: tim_merrigan@nrel.gov• www.nrel.gov

Oak Ridge National Laboratory

Pat M. Love • P.0. Box $2008 \bullet$ One Bethel Valley Road • Oak Ridge, TN $37831 \bullet 865-574-4346 \bullet$ fax: 865-574-9331 • e-mail: lovepm@ornl.gov• www.ornl.gov

Pacific Northwest National Laboratory

Michael C. Baechler • 620 Southwest 5th, Suite $810 \bullet$ Portland, 0R $97204 \bullet 503-417-7553 \bullet$ fax: 503-417-2175•

e-mail: michael.baechler@pnl.gov• www.pnl.gov

Produced for the U.S. Department of Energy (DOE) by the National Renewable Energy Laboratory, a DOE national laboratory. December 2007 - NREL/TP-550-31548

Printed with a renewable-source ink on paper containing at least $50 \%$ wastepaper, including $20 \%$ postconsumer waste. 